\title{
Recomendaciones para el tratamiento y prevención del ictus, 2004
}

\author{
M. Alonso de Leciñana-Cases, G.E. Pérez-R., E. Díez-Tejedor, \\ en representación de la Sociedad Iberoamericana de Enfermedad Cerebrovascular (SIECV)
}

\author{
RECOMMENDATIONS FOR STROKE TREATMENT AND PREVENTION, 2004
}

\begin{abstract}
Summary. Introduction. According to the World Health Organisation, cerebrovascular disease is the third most important cause of death and the leading cause of disability in the adult population in the world today. Yet, stroke patients' progression improves significantly with immediate attention, hospitals that are well equipped with the necessary material and staff (expert neurologists) and the application of protocols as a programme of care, diagnostic methods and specific treatment (stroke units or teams). Development. In order to disseminate the scientific evidence available on stroke treatment and prevention, an ad hoc committee of the Latin-American Society of Cerebrovascular Diseases has drawn up this manual, which includes a summary of all the recommendations currently applicable with the levels of evidence that support them and the degree of recommendation for each case. Conclusions. This document is intended to serve as an aid and a guide to treatment and prevention in stroke patients in all Latin-American countries, and to help to attain the means needed to improve the care given to such patients in the different health care centres. [REV NEUROL 2004; 39: 465-86]
\end{abstract}

Key words. Cerebrovascular disease. Latin-American Society of Cerebrovascular Diseases. Manual. Protocol. Recommendations. Stroke.

\section{INTRODUCCIÓN}

La enfermedad cerebrovascular (ECV) es una de las principales causas de mortalidad y de morbilidad en todo el mundo. Según la OMS, supone la tercera causa de muerte y la primera de invalidez en la población adulta en el mundo. Mientras la mayoría de los países desarrollados muestras tasas estables de mortalidad por ECV, los países en desarrollo incrementan sus tasas anuales de prevalencia y mortalidad; 4,5 millones de los 10 millones de muertos anuales por ECV pertenecen a los países no industrializados. Hasta un tercio de los pacientes que sobreviven quedan con secuelas invalidantes, y hasta un $25 \%$ presentarán después del ictus un deterioro cognitivo en mayor o menor grado [1-4]. Tomando datos de nuestros países en Iberoamérica, diremos, por ejemplo, que en España es la primera causa de muerte en las mujeres y la segunda en los hombres y supone el $10 \%$ de la mortalidad global [5]. El ictus tiene una alta incidencia y prevalencia. Disponemos de datos de algunos países de América del Sur y de la península Ibérica. En el primer caso, la incidencia bruta anual oscila entre los 35 y los 183/100.000 habitantes/año y la prevalencia entre los 174 y los 651/100.000 habitantes [6],

Recibido: 16.07.04. Aceptado: 23.07.04.

Coordinadores: M. Alonso de Leciñana, G.E. Pérez-R., E. Díez-Tejedor. Comité de redacción: M. Alonso de Leciñana (Ávila, España), O. Benavente (San Antonio, EE. UU.), M. Carvajal, F. Chávez-Sell (San José, Costa Rica), E. Díez-Tejedor, J.A. Egido, B. Fuentes (Madrid, España), J.M. Lainez (Valencia, España), M.T. Medina (Tegucigalpa, Honduras), G.E. Pérez-R. (Bogotá, Colombia), G. Saposnik (Buenos Aires, Argentina), J. Tejada (León, España), J. Vivancos (Madrid, España). Comité ad hoc: J. Álvarez-Sabín (Barcelona, España), O. del Brutto (Guayaquil, Ecuador), J. Gállego (Pamplona, España), A. Gil (Madrid, España), A. Gil-Peralta (Sevilla, España), O. Hernández (Curitiba, Brasil), J. Larracoechea (Bilbao, España), R. Leira (Santiago de Compostela, España), J. Maestre (Granada, España), J. Martí-Fábregas (Barcelona, España), E. MartínezVila (Pamplona, España), J. Matías-Guiu (Alicante, España), J. Nader (México DF, México), A. Pareja (Valencia, España), A. Robles (Santo Domingo, Rep. Dominicana), F. Rubio, L. Soler, N. Vila (Barcelona, España).

Correspondencia: Dra. María Alonso de Leciñana. Unidad de Investigación Cerebrovascular. Hospital Universitario La Paz. Paseo de la Castellana, 261.E-28046 Madrid.E-mail: malonso@hnss.sacyl.es

(C) 2004, REVISTA DE NEUROLOGÍA mientras que en España los datos de incidencia oscilan alrededor de 175-200 casos/100.000/año y los de prevalencia en torno a 4.012-7.100/100.000 [7-10]. A pesar de las diferencias en las cifras, que pueden deberse a factores socieconómicos y genéticos, a diferente exposición a factores de riesgo o a la escasez de estudios epidemiológicos en algunos países, es cierto que el ictus supone un auténtico problema de salud que obliga a establecer las mejores pautas de prevención y tratamiento para reducir la incidencia y las secuelas. Además, teniendo en cuenta que la incidencia aumenta en personas mayores de 65 años y que, debido a la mejora en la calidad de vida, se está produciendo un incremento notable de la esperanza de vida y un envejecimiento progresivo de la población mundial, la prevalencia de esta enfermedad aumenta y, consecuentemente, también lo hace la magnitud del problema sociosanitario que supone.

Hay suficientes evidencias que indican que la atención urgente del paciente con ictus, en un medio hospitalario bien dotado de los medios materiales y personales (neurólogos expertos) necesarios para poder aplicar un programa protocolizado de cuidados, métodos diagnósticos y tratamiento específico (unidades de ictus o en su defecto equipos de ictus), mejora significativamente la evolución de los pacientes afectados [11-13].

Con el fin de colaborar en la difusión de las evidencias científicas disponibles acerca del tratamiento y la prevención del ictus, un comité ad hoc de la Sociedad Iberoamericana de Enfermedad Cerebrovascular ha redactado la guía que a continuación se expone. El germen de esta guía se presentó en el VI Congreso Iberoamericano de Enfermedad Cerebrovascular celebrado en San José de Costa Rica en octubre de 2003 y el texto definitivo y actualizado se ha dado a conocer en el VII Congreso celebrado en Cancún, México. En ella se recogen de manera esquemática todas las recomendaciones vigentes, con los niveles de evidencia que las respaldan y el grado de recomendación para cada caso [14] (Tabla I).

Es el deseo de los autores que este documento, expresión de las recomendaciones de la Sociedad Iberoamericana de Enfermedad Cerebrovascular, sirva efectivamente de apoyo y guía para el tratamiento y la prevención en los pacientes con ictus en todos los países de Iberoamérica y que contribuya también a 
Tabla I. Niveles de evidencia y grados de recomendación.

Nivel de evidencia I: grandes estudios aleatorizados con poco riesgo de error, tanto para falsos positivos $(\alpha)$ como para falsos negativos $(\beta)$

Nivel de evidencia II: estudios aleatorizados, pero con casuística insuficiente para alcanzar significación estadística y, por tanto, con riesgo de error $\alpha$ o $\beta$, o estudios aleatorizados pero discutibles y con riesgo de error moderado o grande

Nivel de evidencia III: trabajos no aleatorizados, en los que se comparan los pacientes que recibieron o no un tratamiento en la misma época

Nivel de evidencia IV: estudios históricos, no aleatorizados, que comparan resultados entre los pacientes que recibieron o no un tratamiento en diferentes épocas, o los comparan con datos de la bibliografía

Nivel de evidencia V: serie de casos sin controles

Grado A: apoyado al menos por un trabajo, preferiblemente más, del nivel I

Grado B: basado al menos en un trabajo del nivel II

Grado C: apoyado por estudios del nivel III, IV o V

conseguir los medios necesarios para mejorar la atención al paciente con ictus en las distintas administraciones sanitarias.

\section{PREVENCIÓN DE LA ISQUEMIA CEREBRAL}

\subsection{Prevención primaria. Recomendaciones}

\subsubsection{Control de los factores tradicionales}

de riesgo vascular (Tabla II)

- Vigilancia de la presión arterial. En los adultos, como mínimo, a partir de los 40 años y, al menos, cada dos años. Se debe controlar y tratar la hipertensión arterial (HTA) si la presión arterial sistólica (PAS) es superior a $140 \mathrm{mmHg}$ y la presión arterial diastólica (PAD) mayor de $90 \mathrm{mmHg}$, preferiblemente con fármacos inhibidores del enzima convertidor de angiotensina (IECA) (nivel de evidencia I y grado de recomendación A) [1-8].

- Vigilancia y control de la diabetes mellitus. En la actualidad, no hay evidencia suficiente de que el control de la hiperglucemia disminuya per se la incidencia de ictus. Se recomienda mantener un estrecho control de la presión arterial en los pacientes con diabetes mellitus mediante IECA (nivel de evidencia II y grado de recomendación B) [9-10].

- Vigilancia y control del perfil lipídico. Tratar con estatinas a los pacientes con isquemia miocárdica, tengan o no hiperlipidemia, reduce significativamente el riesgo de ictus [11]. Debe tratarse la hiperlipemia en los pacientes con otros factores de riesgo vascular (nivel de evidencia I, grado de recomendación A) [12-16].

- Modificación de hábitos. Deberá suspenderse el consumo de tabaco. Se recomienda reducir el consumo excesivo de alcohol, que no excederá el equivalente a dos copas de vino al día [17].

- Uso de antiagregantes plaquetarios. No se recomienda el uso de aspirina en la prevención primaria del ictus, por cuanto las complicaciones sobrepasan el potencial beneficio (nivel de evidencia I, grado de recomendación A) [18-21].

\subsubsection{Manejo de cardiopatías embolígenas (Tablas III y IV)}

- Fibrilación auricular no valvular (FANV). Se recomienda un
Tabla II. Factores de riesgo de infarto cerebral (modificada de [2])

1. Factores de riesgo bien documentados o confirmados

1.1 Modificables

Hipertensión arterial

Cardiopatía: Fibrilación auricular

Endocarditis infecciosa

Estenosis mitral

Infarto de miocardio reciente

Tabaquismo

Anemia de células falciformes

Ictus o ataque isquémico transitorio previos

Estenosis carotídea asintomática

Hipercolesterolemia

Consumo de alcohol

Inactividad física.

Obesidad

Hematocrito elevado

Factores dietéticos

Hiperinsulinemia y resistencia a la insulina

1.2 Potencialmente modificables

Diabetes mellitus

Homocistinemia

Estados de hipercoagulabilidad

Hipertrofia ventricular izquierda

Infecciones

Migraña

Procesos subclínicos

1.3 No modificables

Edad

Sexo

Factores hereditarios

Etnia

Localización geográfica

Nivel sociocultural

2. Factores de riesgo menos documentados o posibles

2.1 Potencialmente modificables

Cardiopatía: Miocardiopatía

Discinesia de la pared ventricular

Endocarditis no bacteriana

Calcificación del anillo mitral

Estenosis aórtica

Prolapso mitral

Foramen oval permeable

Aneurisma del septo atrial

Contraste ecocardiográfico espontáneo

Uso de anticonceptivos orales

Consumo de drogas

2.2 No modificables

Estación y clima 
Tabla III. Indicaciones sugeridas de la anticoagulación oral en la prevención del infarto cerebral.

Prevención primaria (en las siguientes cardiopatías embolígenas)

\begin{tabular}{l}
\hline Fibrilación auricular $^{a}$ \\
\hline Estenosis mitral \\
\hline Síndrótesis valvular $^{\text {Miocardiopatía dilatada }}$ \\
\hline Infarto de miocardio reciente con trombo ventricular izquierdo \\
\hline Prolapso de la válvula mitral ${ }^{\text {a }}$ \\
Prevención secundaria
\end{tabular}

\begin{tabular}{l}
\hline Cardiopatías embolígenas descritas previamente \\
\hline Estados protrombóticos \\
$\frac{\text { Déficit de proteína } \mathrm{C} \text {, proteína } \mathrm{S} \text {, antitrombina III }}{\text { Síndrome antifosfolípido }^{\mathrm{a}}}$ \\
Mutación del factor $\mathrm{V}$ de Leyden $^{\mathrm{a}}$
\end{tabular}

Infarto criptogénico recurrente (si falla el tratamiento antiagregante) ${ }^{b}$

Placa de ateroma en el cayado aórtico ${ }^{\text {b }}$

Lesión arterial inducida por radiación ${ }^{\text {b }}$

Foramen oval permeable ${ }^{a}$

Aneurisma del septo auricular ${ }^{a}$

Trombo en el ventrículo izquierdo

Disección arterial (cervical y cerebral) ${ }^{b}$

${ }^{a}$ Indicado en pacientes de alto riesgo. ${ }^{b}$ Utilidad controvertida. Modificada de [44].

tratamiento anticoagulante con cumarínicos y un INR 2-3, excepto en los menores de 65 años y sin otros factores de riesgo, en quienes se recomienda 300 mg/día de ácido acetilsalicílico (AAS). En los que esté contraindicada la anticoagulación se aconseja antiagregación con $300 \mathrm{mg} /$ día de AAS (nivel de evidencia I y grado de recomendación A) [22-31].

- Valvulopatía mitral reumática. Está indicada la anticoagulación oral (INR 2-3), en particular si concurre con una o más de las siguientes circunstancias: fibrilación auricular (FA) crónica o paroxística, embolismo sistémico previo. También se recomienda la anticoagulación cuando la aurícula es mayor de $55 \mathrm{~mm}$ aun en ritmo sinusal [22-23].

- Prótesis mecánicas. Está indicado el tratamiento anticoagulante oral, inicialmente con INR 2,5-3,5, para posteriormente ajustar el nivel de anticoagulación en función de la localización y modelo de la válvula así como de la presencia de factores de riesgo. En los pacientes con bioprótesis y sin otros factores de riesgo se aconseja anticoagulación oral durante los primeros tres meses tras la implantación con INR 2-3 y luego $300 \mathrm{mg} /$ día de aspirina (nivel de evidencia II y grado de recomendación B) [22-23].

- Cardiopatía isquémica. En los pacientes con infarto agudo de miocardio (IAM) y alto riesgo de embolismo sistémico (IAM anterior y extenso, aneurisma ventricular izquierdo, presencia de trombo intraventricular y embolismo previo) se recomienda administrar heparina IV en dosis terapéuti- cas durante 7-10 días (TPTA 1,5-2) y continuar con anticoagulantes orales (INR 2-3) tres meses. Sólo en caso de dilatación del ventrículo izquierdo, discinesia ventricular, fracción de eyección menor del $30 \%$ o insuficiencia cardíaca se recomienda la anticoagulación a largo plazo. (nivel de evidencia I y grado de recomendación A) [22-23].

- Miocardiopatía dilatada. En los pacientes con una fracción de eyección menor al 30\% y sin otros factores de riesgo o en ausencia de embolismo previo se aconseja $300 \mathrm{mg} /$ día de AAS y anticoagulación oral (INR 2-3) si la fracción de eyección es menor al $30 \%$ y hay FA, embolismo previo o trombo intraventricular [22-23].

- Prolapso de la válvula mitral. En pacientes menores de 65 años y en ritmo sinusal se recomienda 300 mg/día de AAS; en los mayores de 65 años está indicada la anticoagulación con INR 2-3 si se asocian FA, insuficiencia mitral o insuficiencia cardíaca [22-23].

- Foramen oval permeable o aneurisma de fosa oval. Se recomienda iniciar tratamiento con $300 \mathrm{mg} /$ día de AAS (nivel de evidencia II y grado de recomendación B) [22-23].

\subsubsection{Manejo de la estenosis carotídea asintomática}

Cuando la lesión es mayor del $60 \%$, se aconseja la evaluación neurológica, el control de los factores de riesgo asociados y, en casos seleccionados, considerar la posibilidad de endarterectomía, que se podría indicar en pacientes con estenosis del 60 al $99 \%$ con bajo riesgo quirúrgico (menor al $3 \%$ ) y expectativa de vida superior a cinco años (nivel de evidencia I y grado de recomendación A) [32-39].

\subsection{Prevención secundaria. Recomendaciones}

\subsubsection{Control de los factores tradicionales de riesgo vascular}

- Tratamiento de la HTA. Tratar y controlar la hipertensión arterial en pacientes con antecedentes de ictus es el pilar de la prevención secundaria Se recomienda mantener valolres inferiores a 135/85. Los datos disponibles de estudios aleatorizados en prevención secundaria indican que IECA o la combinación de IECA y diurético es la mejor opción en estos pacientes (nivel de evidencia I y grado de recomendación A) [40-53].

- Perfil lipídico. Los pacientes con ictus y antecedentes de cardiopatía isquémica deben tratarse con estatinas independientemente de la concentración de colesterol. En la isquemia cerebral asociada a enfermedad ateroesclerótica y concentración de LDL igual o superior a $130 \mathrm{mg} / \mathrm{dL}$ se iniciará el tratamiento con estatinas (la meta meta es menos de $100 \mathrm{mg}$ de LDL). El resto de los pacientes con ictus, dependerá del riesgo vascular, según el National Cholesterol Education Program (nivel de evidencia I y grado de recomendación A) [54-60].

- Modificación de hábitos. Se mantienen las recomendaciones de suspender el tabaquismo y moderar el consumo de alcohol.

\subsubsection{Prevención secundaria de la isquemia}

cerebral asociada a arterioesclerosis de gran vaso

y enfermedad de pequeño vaso

- Uso de antiagregantes. Se recomienda la antiagregación oral en la prevención secundaria de la isquemia cerebral (IC) usando 100-300 mg/día de AAS, 75 mg/día de clopidogrel o $300 \mathrm{mg} / 12 \mathrm{~h}$ de triflusal como primera elección o alternativa según el riesgo vascular, los efectos adversos o las recurrencias. En los países en los que está disponible, se 
M. ALONSO DE LECIÑANA-CASES, ET AL

Tabla IV. Prevención del embolismo cerebral de origen cardíaco.

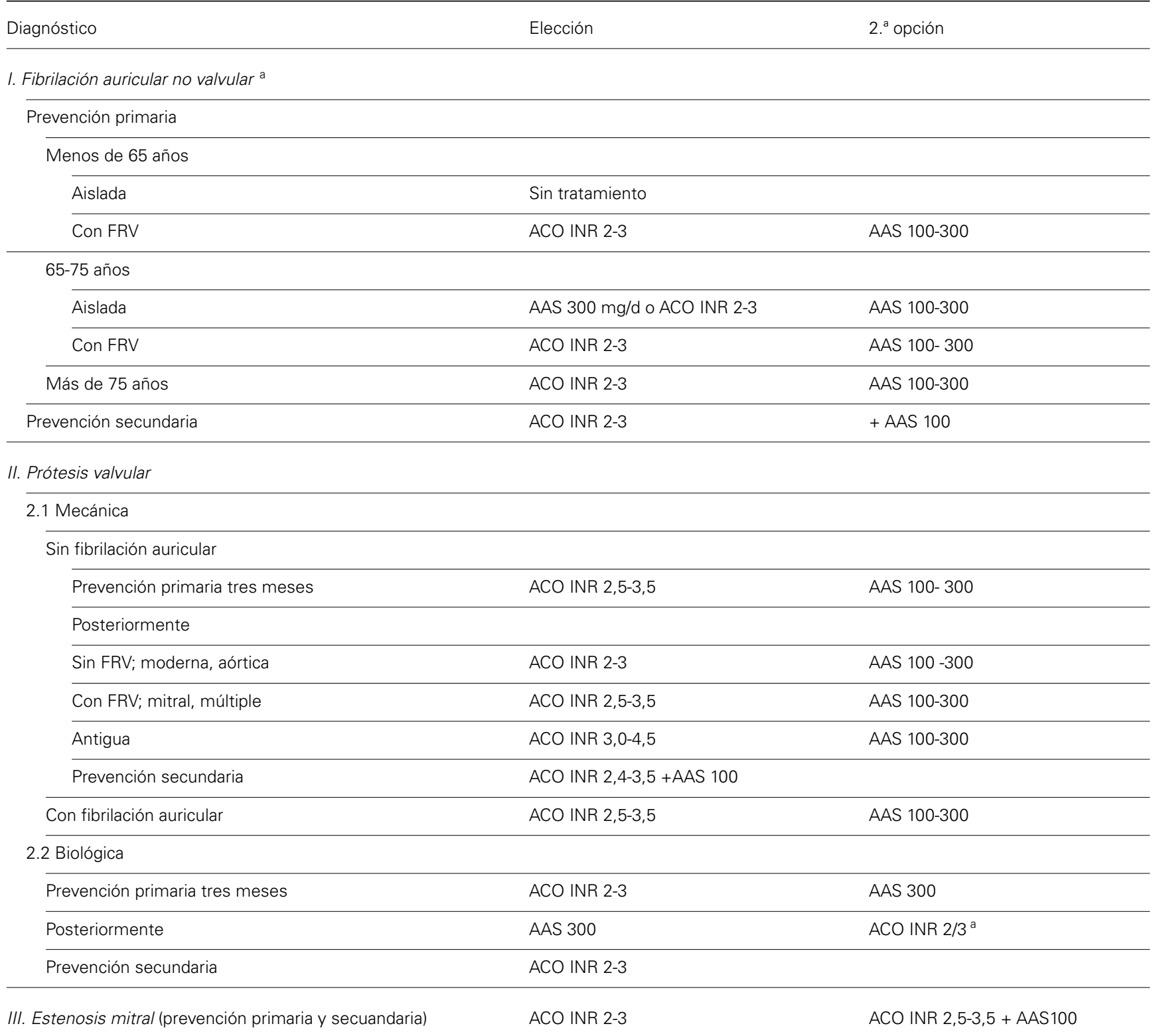

\section{Prolapso mitral}

Prevención primaria

Menos de 65 años, sin signos ecocardiográficos de gravedad y ritmo sinusal

AAS 300

Más de 65 años y fibrilación auricular,

insuficiencia mitral, insuficiencia cardíaca

Prevención secundaria

Menos de 65 años

AIT, fibrilación auricular sin insuficencia mitral, HTA, o IC

or

Ictus previo ACO INR 2-3

Ictus previo y contraindicación de ACO AAS 300

Más de 65 años 
Tabla IV. Prevención de embolismo cerebral de origen cardíaco (cont.).

\begin{tabular}{|c|c|c|}
\hline Diagnóstico & Elección & 2. apción \\
\hline \multicolumn{3}{|l|}{ V. Cardiopatía isquémica } \\
\hline \multicolumn{3}{|l|}{ IAM (prevención primaria y secuandaria) } \\
\hline Menos de cuatro semanas & Heparina iv. (TPTA 1,5-2) & Heparina BPM o AAS 100/300 \\
\hline $\begin{array}{l}\text { Extenso, anterior, trombo } \\
\text { intraventricular, embolismo periférico }\end{array}$ & ACO INR 2-3 (3 meses) & \\
\hline Aneurisma ventricular (prevención primaria y secuandaria) & ACO INR 2-3 & AAS 100- 300 \\
\hline \multicolumn{3}{|l|}{ Aquinesia segmentaria de $\mathrm{VI}$} \\
\hline Prevención primaria & AAS 300 & \\
\hline Prevención secundaria & ACO INR 2-3 & AAS 100-300 \\
\hline \multicolumn{3}{|l|}{ VI. Miocardiopatía dilatada } \\
\hline \multicolumn{3}{|l|}{ Prevención primaria } \\
\hline $\begin{array}{l}\text { Fracción de eyección menor al } 30 \% \text {, isquemia } \\
\text { sin fibrilación auricular ni otros factores de riesgo }\end{array}$ & AAS 300 & \\
\hline $\begin{array}{l}\text { Fracción de eyección menor al } 30 \% \text { y } \\
\text { fibrilación auricular, embolismo previo, trombo VI }\end{array}$ & ACO INR 2-3 & AAS $100-300$ \\
\hline Prevención secundaria & ACO INR 2-3 & \\
\hline VII. Mixoma auricular (prevención primaria y secuandaria) & ACO INR 2-3 & AAS 100-300 \\
\hline \multicolumn{3}{|l|}{ VIII. Foramen oval permeable/aneurisma de fosa oval } \\
\hline Prevención primaria & AAS 300 & \\
\hline Prevención secundaria & ACO INR 2-3 & AAS $100-300$ \\
\hline
\end{tabular}

puede indicar la combinación de aspirina $(25 \mathrm{mg}$ ) y dipiridamol (200 mg) dos veces al día. La evidencia disponible sugiere que los pacientes con alto riesgo de recidiva (mayor del $5 \%$ por año) obtienen un beneficio adicional del clopidogrel frente a la aspirina (nivel de evidencia I y grado de recomendación A) [61-76].

No existen datos para recomendar la anticoagulación oral en la prevención secundaria de los pacientes con IC de origen aterotrombótico [77-79].

- Enfermedad carotídea sintomática. Se recomienda la endarterectomía carotídea en los pacientes menores de 80 años con estenosis carotídea ipsilateral del $70-99 \%$ que hayan presentado accidente isquémico transitorio (AIT), amaurosis fugaz o infarto cerebral con secuelas menores. La tasa de morbimortalidad perioperatoria debe ser inferior al 6\%. La endarterectomía podría indicarse en pacientes varones con estenosis entre el $50 \mathrm{y}$ el $69 \%$, con alto riesgo y si el riesgo quirúrgico es inferior al 3\%. Las estenosis inferiores no deben someterse a este tratamiento (nivel de evidencia I y grado de recomendación A) [80-84]. La angioplastia transluminal percutánea debe realizarse en los casos de estenosis carotídea superior al $70 \%$ en los que exista contraindicación para la endarterectomía, dificultades técnicas, reestenosis tras endarterectomía, estenosis posradioterapia o displasia fibrosa. Es una opción en estenosis marcadas carotídeas altas, de arterias intracraneales y vertebrales (nivel de evidencia I y grado de recomendación A) $[85,86]$.

\subsubsection{Tratamiento farmacológico en la prevención secundaria} de la isquemia cerebral de origen cardioembólico (Tablas III y IV)

Se recomienda la anticoagulación oral (INR 2-3) en los pacientes con fibrilación auricular que hayan presentado IC (nivel de evidencia I y grado de recomendación A) [22-23,87].

En pacientes con valvulopatía reumática y embolismos recurrentes se aconseja mantener INR en el límite superior (2,5-3),o bien asociar $100 \mathrm{mg} /$ día de AAS [22].

En las prótesis mecánicas con episodios embólicos está indicada la anticoagulación con INR en el límite superior (2,5-3) o las asociación de $100 \mathrm{mg}$ /día de AAS [22].

En los pacientes con antecedentes de infarto de miocardio e IC deberá considerarse la anticoagulación oral permanente si existe dilatación y disfunción del ventrículo izquierdo [22].

En el prolapso mitral con AIT previo se recomienda antiagregación con aspirina (100 mg/día). Si hay recurrencias, anticoagulación oral INR 2-3 [22].

En el foramen oval permeable o aneurisma de fosa oval está 


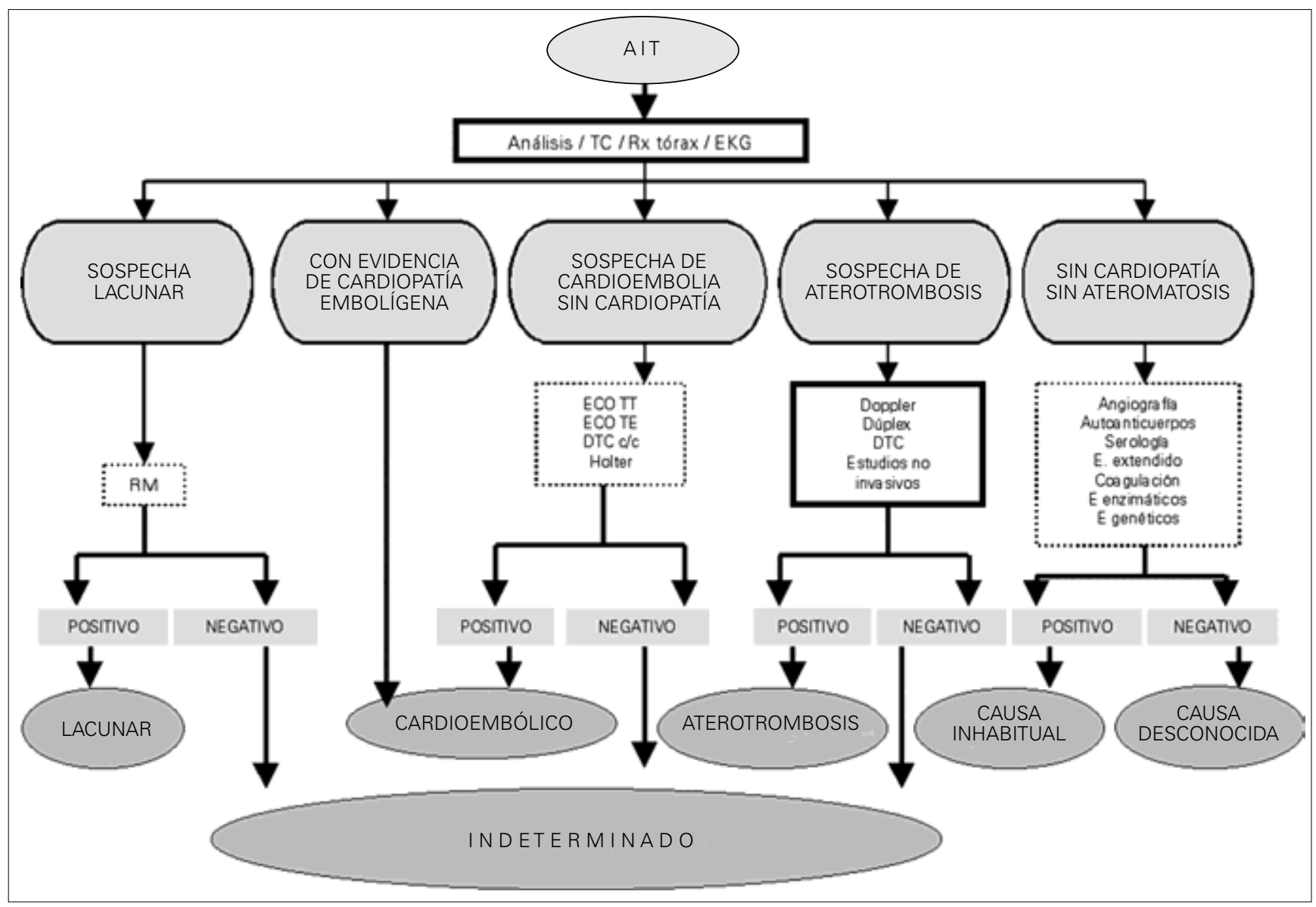

Figura 1. Algoritmo diagnóstico del ataque isquémico transitorio.

indicado el tratamiento anticoagulante (INR 2-3) en los pacientes que han presentado una manifestación embólica. El cierre quirúrgico deberá considerarse en pacientes con riesgo de

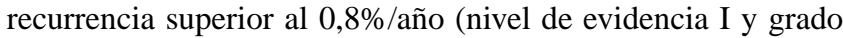
de recomendación A) [88-94].

\subsubsection{Tratamiento farmacológico en la prevención} secundaria de la isquemia cerebral de causa inhabitual

- Disección arterial. Se recomienda antiagregación plaquetaria o anticoagulación, inicialmente con heparina sódica y posteriormente con anticoagulantes orales durante tres meses (nivel de evidencia III y grado de recomendación C) [95-97].

- Déficit de proteína C, S y antitrombina III, anticoagulante lúpico. En la prevención secundaria se indica el tratamiento anticoagulante (nivel de evidencia III y grado de recomendación C) [98,99].

- Placas de ateroma en el cayado aórtico, dolicoestasia de la arteria basilar, estenosis intracraneal. Se recomienda en principio antiagregación plaquetaria, aunque en algunos casos podría resultar útil el tratamiento anticoagulante [100-101].

\subsubsection{Tratamiento farmacológico en la prevención secundaria} de la isquemia cerebral de origen indeterminado

En el infarto de origen indeterminado se recomienda completar el estudio, y si se identifica más de una etiología, tratar al menos la de mayor riesgo de recurrencia. En caso de ser criptogénico, se usan antiagregantes.

\section{ACTUACIÓN CLÍNICA EN EL ATAQUE ISQUÉMICO TRANSITORIO}

3.1. Evaluación clínica y diagnóstico diferencial. Recomendaciones

Debe realizarse una anamnesis detallada para determinar las características y la duración del episodio y distinguirlo de otros eventos que no deben considerarse AIT (Tabla V) [1-7].

Los estudios complementarios en el AIT deben seguir el algoritmo que se indica en la figura 1, en función de sus características y de la sospecha clínica.

Se recomienda hospitalización para realizar el estudio que permita diagnosticar la etiología, evaluar el riesgo de recurrencia e indicar un tratamiento específico. Una vez hecha la evaluación general en urgencias, un neurólogo ha de evaluar a los pacientes con AIT lo antes posible [8-51].

\subsection{Prevención. Recomendaciones}

\subsubsection{Control de los factores de riesgo}

La HTA debe tratarse para mantener una presión sistólica por debajo de $140 \mathrm{mmHg}$ y una presión diastólica por debajo de 90 $\mathrm{mmHg}$. Para diabéticos la presión arterial debe de estar por debajo de 130/85 mmHg [51-56].

Dejar de fumar completamente [57].

Controla y tratar adecuadamente las enfermedades cardíacas (cardiopatía isquémica, insuficiencia cardíaca, arritmias y valvulopatías) [33,51].

Suprimir el consumo excesivo de alcohol. Se permiten una o dos bebidas al día [58]. 
Tabla V. Guía para el diagnóstico de AIT en urgencias.

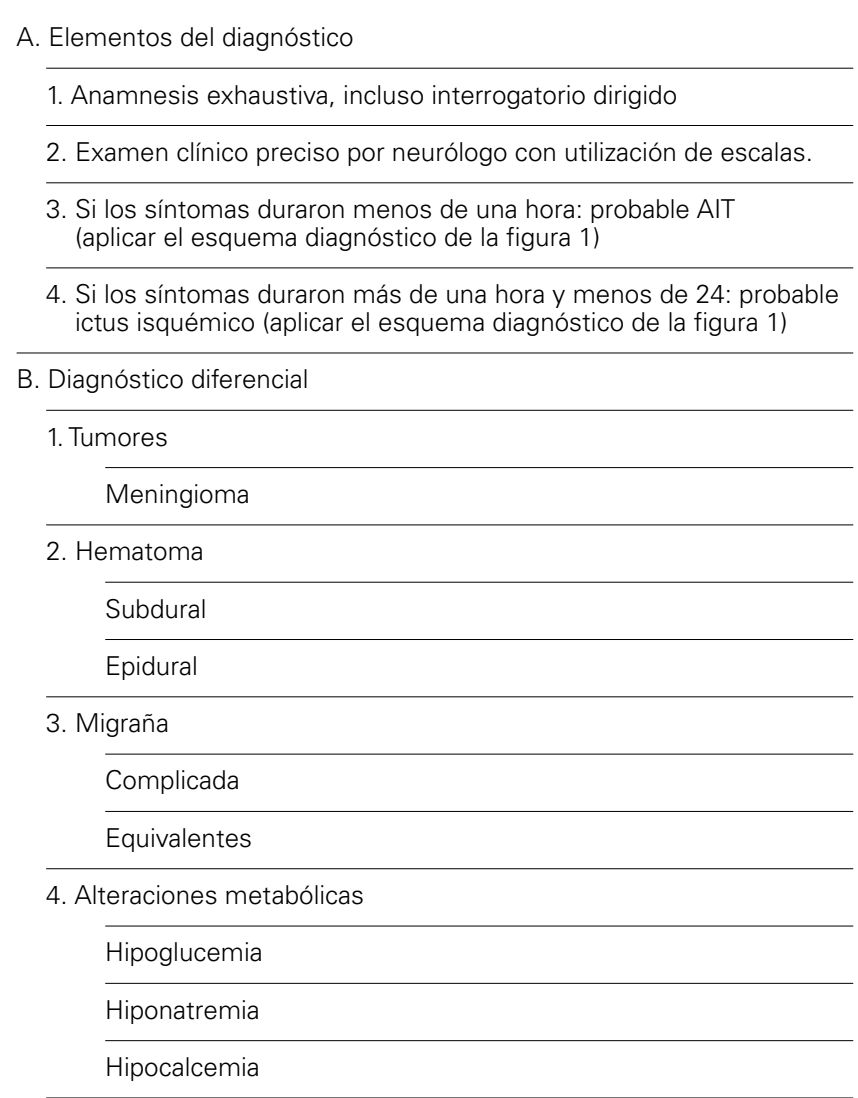

5. Síncope
6. Crisis convulsivas
Inhibitorias

7. Brote agudo de esclerosis múltiple

8. Otros

Enfermedad vestibular

Narcolepsia

Hiperventilación

Vigilar y tratar el perfil lipídico. Inicialmente, con medidas higienicodietéticas: dieta hipolipemiante y una actividad física apropiada. Si después de realizar esto el nivel de LDL se mantiene por encima de $130 \mathrm{mg} / \mathrm{dL}$, se deben utilizar las estatinas. $\mathrm{El}$ objetivo, en quienes han presentado un AIT, es mantener una concentración de LDL inferior a $100 \mathrm{mg} / \mathrm{dL}$ [59-62].

La concentración de glucosa en ayunas no debe exceder los $120 \mathrm{mg} / \mathrm{dL}$. Se utilizarán dietas, antidiabéticos orales o insulina, hasta que se obtenga una concentración adecuada [63,64].

Se aconseja realizar actividad física, de 30 a 60 minutos, tres o cuatro veces a la semana. El paciente debe recibir instrucción para utilizar al menos el $65 \%$ de su frecuencia cardíaca máxima (o de fatiga) [58].

No se deben utilizar anticonceptivos orales con altas dosis de estrógenos, y en todo caso evitar su uso en mujeres de más de 35 años que sean migrañosas, fumadoras o que tengan algún factor de riesgo cardiovascular. Los últimos estudios no recomiendan de forma general la terapia sustitutiva con estrógenos en la posmenopausia [65,66] (nivel de evidencia I y grado A).

\subsubsection{Tratamiento médico}

- Ataques isquémicos transitorios de origen aterotrombótico. Todos los pacientes que han tenido un AIT aterotrombótico deben recibir un antiagregante plaquetario para reducir el riesgo de recurrencia. Se recomienda AAS en dosis de $100 \mathrm{mg} /$ día. En caso de contraindicación, efecto secundario o recurrencias se pueden utilizar otros antiagregantes, como clopidogrel (75 mg/día) o triflusal (300 mg/12 h) [67-74] (nivel I-II y grado A). En los pacientes de alto riesgo es preferible utilizar clopidogrel en dosis de $75 \mathrm{mg} /$ día.

El tratamiento anticoagulante en los pacientes con AIT aterotrombóticos sólo se recomienda en los que tienen nuevos AIT del mismo territorio, a pesar de recibir tratamiento con antiagregantes plaquetarios. El nivel de anticoagulación debe controlarse con frecuencia, debido a que un INR de más de 3,0 se asocia con un mayor riesgo de hemorragias [75,76].

- Ataques isquémicos transitorios de origen cardíaco. Está indicada la anticoagulación a largo plazo en aquellos enfermos que presentan fibrilación auricular. El INR debe estar alrededor de 2,5 (2 a 3) (nivel I y grado A). La aspirina sólo se recomienda cuando existe una contraindicación real a la anticoagulación en dosis de $300 \mathrm{mg} /$ día. La anticoagulación oral podría estar indicada en pacientes con otras fuentes de cardioembolismo (ver capítulo de prevención) $[77,78]$.

\subsubsection{Tratamiento quirúrgico}

La endarterectomía carotídea o angioplastia está indicada en pacientes con AIT ispilateral, reciente (en los seis meses previos) y estenosis carotídea extracraneal entre el 70 y el $99 \%$, después de una cuidadosa selección y en centros acreditados con una morbimortalidad perioperatoria inferior al 7\% (nivel I y grado A) [79-90].

La endarterectomía en pacientes con AIT ipsilateral reciente (en los seis meses previos) y estenosis carotídea extracraneal entre el 50 y $69 \%$ ofrece un margen de beneficio inferior, pero podría contemplarse en centros con una morbimortalidad perioperatoria inferior al 3\% (nivel I y grado A).

La angioplastia carotídea es una buena alternativa a la endarterectomía (nivel I y grado A) [91,92].

\section{MANEJO DEL INFARTO CEREBRAL AGUDO 4.1. Sistemática asistencial. Recomendaciones}

La atención al paciente con infarto cerebral debe hacerse de manera urgente y protocolizada, preferiblemente por expertos en el manejo de estos procesos, con el fin de llegar a un diagnóstico etiopatogénico correcto y poder aplicar el tratamiento adecuado para reducir la mortalidad y las secuelas que produce esta enfermedad [1-4].

Todos los pacientes con ictus agudo deben atenderse con carácter de urgencia en el medio hospitalario [4-7] (grado C).

Se recomienda la evaluación precoz por un especialista en neurología [5] (grado C).

Se recomienda el ingreso en unidades de ictus agudo con la dotación necesaria [8-10] (grado A). 
Tabla VI. Sistemática diagnóstica en el ictus agudo.

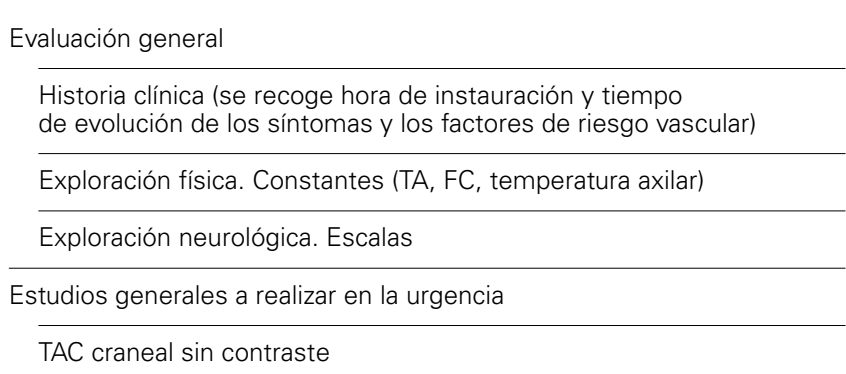

Electrocardiograma

Radiografía del tórax

Estudio hematológico (recuento celular, plaquetas)

Estudio de coagulación (INR, actividad de protrombina, tiempo parcial de tromboplastina activada)

Bioquímica (glucosa, iones, función renal, función hepática)

Otras en función de la clínica (gasometría arterial,

punción lumbar en sospecha de subaracnoidea)

Estudios específicos dirigidos a definir la etiología

Estudio neurovascular no invasivo (Doppler carotídeo y transcraneal)

Estudio cardiológico: ecocardiograma

(transtorácico/transesofágico), Holter

Arteriografía

Estudio inmunológico, serológico (sangre, LCR)

Estudio hematológico para detectar estados procoagulantes

Otros

En ausencia de unidades de ictus se recomienda la atención por equipos de ictus [11] (grado B).

Los hospitales que atiendan pacientes con ictus deben estar dotados de los medios técnicos y de personal necesarios para ello (servicio de urgencias, tomografía axial computarizada -TAC- las 24 horas, laboratorio las 24 horas, neurólogo las 24 horas, unidades de ictus, equipos de ictus) [1-4] (grado C).

\subsection{Sistemática diagnóstica. Recomendaciones}

Se recomienda realizar las técnicas diagnósticas que se resumen en la tabla VI. Los objetivos son, inicialmente, definir la situación clínica y, posteriormente, la etiología del cuadro. Todas las actuaciones deben realizarse de forma protocolizada y en el menor tiempo posible.

\subsection{Medidas generales de tratamiento. Recomendaciones}

Se recomienda monitorizar la saturación de $\mathrm{O}_{2}$ mediante pulsioximetría en todos los pacientes con ictus isquémico (grado $\mathrm{C}$ ).

Si la pulsioximetría muestra una saturación de $\mathrm{O}_{2}$ inferior al 95\% o la gasometría arterial revela una hipoxemia franca se administrará oxigenoterapia a $3 \mathrm{~L} / \mathrm{min}[2,3]$ (grado C). No se recomienda generalizar a todos los pacientes el aporte suplementario de oxígeno [12] (grado B).

Se recomienda intubación y soporte ventilatorio a los pacientes con afectación de la vía aérea [13] (grado C).

Se monitorizará la tensión arterial con tomas, al menos cada cuatro horas o más frecuentemente en caso necesario.
Tabla VII. Recomendaciones para el tratamiento de la tensión arterial en el ictus agudo.

\begin{tabular}{|c|c|}
\hline Fase 1 & $\begin{array}{l}\text { Control de situaciones que pueden causar hipertensión } \\
\text { (globo vesical, dolor, fiebre, hipoxia, hipertensión endocraneal) }\end{array}$ \\
\hline Fase 2 & $\begin{array}{l}\text { Labetalol ( } 10-20 \mathrm{mg} \text { en bolo en } 1-2 \text { minutos repetidos cada } \\
10 \text { minutos hasta respuesta o dosis máxima de } 300 \mathrm{mg} \text { o } \\
\text { bien seguir el bolo inicial de perfusión continua } 2-8 \mathrm{mg} / \mathrm{min} \text { ) } \\
\text { o } \\
\text { Enalapril }(1-5 \mathrm{mg} / 6 \mathrm{~h}) \\
\text { O } \\
\text { Urapidil }(10-50 \mathrm{mg} / 6-8 \mathrm{~h})\end{array}$ \\
\hline Fase 3 & $\begin{array}{l}\text { Nitroprusiato }(0,15 \mu \mathrm{g} / \mathrm{kg} / \mathrm{min} \text { en } \\
\text { perfusión hasta el control de la TA })\end{array}$ \\
\hline
\end{tabular}

En la mayoría de los pacientes, la hipertensión arterial no debe recibir tratamiento, pues es un fenómeno reactivo que tiende a la normalización [14-16] (grado C).

Se recomienda tratar con fármacos antihipertensivos si la tensión arterial sistólica es mayor de $220 \mathrm{mmHg}$ o la diastólica mayor de $120 \mathrm{mmHg}$ [1-4], aunque ésta es una recomendación de consenso, pues no hay ensayos clínicos que la avalen con un alto nivel de evidencia. Si el paciente va a recibir trombólisis, el límite permitido de TA es 185/110, para reducir el riesgo de hemorragia cerebral $[17,18]$. En términos generales, ésta es la cifra que se recomienda para todos los pacientes $[15,16]$. Se usarán fármacos con efecto predecible y no brusco, por vía oral o intravenosa y que no reduzcan la presión de perfusión, a fin de preservar el flujo en el área de penumbra $[1,4]$. Por este motivo, en la fase aguda no deben usarse antagonistas del calcio (grado C) (Tabla VII).

Algunas situaciones pueden requerir tratamiento hipotensor urgente (encefalopatía hipertensiva, infarto agudo de miocardio, disección aórtica, edema agudo de pulmón).

Cuando exista hipotensión, se recomienda investigar las causas y corregir con expansores de plasma y dopamina si fallan los primeros (grado C)

La temperatura se tomará cada seis horas. Puesto que la fiebre se asocia al incremento de la morbilidad y la mortalidad en los pacientes con ictus agudo, se recomienda identificar y tratar la causa; pueden usarse antipiréticos (paracetamol) o medidas físicas en los pacientes con una temperatura axilar superior a $37,5^{\circ} \mathrm{C}[18,19]$ (grado A).

Se recomienda el tratamiento de la hiperglucemia o hipoglucemia durante la fase aguda del ictus, procurando mantener al paciente normoglucémico [21]. No deben administrarse soluciones glucosadas al $5 \%$ puesto que son hipotónicas; cuando exista hipoglucemia deberán usarse soluciones glucosadas al 33 o el $50 \%$ por vía venosa (grado C). La hiperglucemia (por encima de $180-200 \mathrm{mg} / \mathrm{dL}$ ) se tratará mediante la administración de insulina (grado B) [22].

Debe evaluarse la presencia de disfagia para prevenir la posibilidad de broncoaspiración, y proceder a la alimentación enteral mediante sonda nasogástrica en caso necesario [23] (grado C). Si se prevé la necesidad de administrar la nutrición por vía enteral de manera prolongada, es preferible colocar una sonda de gastrostomía (grado B) [24].

Se recomienda evitar la desnutrición, puesto que puede interferir con la recuperación tras el ictus [25] y, en caso de imposibilidad para la deglución, considerar la necesidad de alimentación enteral tras las primeras $24-48$ horas (grado C).

Se recomienda la movilización en las primeras 24 horas (cambios posturales, movilización pasiva de las extremidades, 
sedestación) junto con un adecuado cuidado de la piel para prevenir complicaciones de la inmovilidad (trombosis venosas, úlceras de decúbito, anquilosis, contracturas, parálisis por presión, neumonía) [26] (grado C).

Se recomienda el empleo precoz de técnicas de fisioterapia y rehabilitación para mejorar la recuperación funcional (grado A) [26-30].

\subsection{Prevención y tratamiento de las complicaciones neurológicas. Recomendaciones}

\subsubsection{Edema cerebral, hipertensión endocraneal e hidrocefalia}

El primer paso es evitar situaciones que puedan agravar el edema cerebral y la hipertensión endocraneal secundaria. Se aconseja una ligera restricción de líquidos, evitar el uso de soluciones hipotónicas (por ejemplo, glucosa al 5\%) y corregir situaciones de hipoxia, hipercarbia o hipertermia (grado de recomendación C) [31,32]. La cabecera de la cama debe elevarse 20-30. Se evitará el uso de hipotensores, especialmente de vasodilatadores (grado de recomendación C) [1,2].

Los corticoides o los barbitúricos no deben emplearse en el tratamiento farmacológico del edema cerebral de causa isquémica (grado de recomendación A) [32-35].

Si la situación clínica se deteriora debido al edema cerebral se recomienda osmoterapia e hiperventilación (grado B). Se recomienda osmoterapia con manitol al $20 \%(0,25-0,5 \mathrm{~g} / \mathrm{kg} / 6 \mathrm{~h})$ [36] como primera elección o bien glicerol al 10\% [37] (grado C). La osmolaridad plasmática debe mantenerse por debajo de $320 \mathrm{mOsm} / \mathrm{L}$. La hiperventilación mediante intubación para procurar una caída de la $\mathrm{PCO}_{2}$ de 5-10 mmHg debe complementarse con otras medidas para el control permanente de la hipertensión endocraneal (grado C) [2].

Se recomienda el drenaje ventricular en los pacientes con hipertensión intracraneal debida a hidrocefalia (grado A). En pacientes seleccionados con infartos cerebrales malignos puede estar indicada la descompresión quirúrgica por craniectomía, aunque las secuelas de la intervención pueden ser muy importantes [38].

Se recomienda la craniectomía suboccipital descompresiva o la ventriculostomía para liberar la hidrocefalia y la compresión del tronco del encéfalo secundarias a infartos cerebelosos [39] (grado C).

\subsubsection{Crisis comiciales}

Pueden producirse crisis comiciales en algunos casos tras un ictus, especialmente en las primeras 24 horas, sin que ello tenga repercusión sobre la evolución clínica. No se recomienda tratar las crisis aisladas con fármacos antiepilépticos, pero sí las recurrentes o tardías, siguiendo las recomendaciones generales del tratamiento de las crisis epilépticas de cualquier causa [1,40,41].

El estado epiléptico debe tratarse con fenitoína $(15-18 \mathrm{mg} / \mathrm{kg}$ disueltos en suero salino que se pasa IV en dos horas como dosis de carga y con monitorización cardíaca,) o ácido valproico $(15 \mathrm{mg} / \mathrm{kg}$ en bolo en cinco minutos y posteriormente perfusión intravenosa de $1 \mathrm{mg} / \mathrm{kg} / \mathrm{h}$ ). Si no se consigue el control debe instaurarse tratamiento en la UCI con barbitúricos.

\subsection{Prevención y tratamiento de las complicaciones no neurológicas. Recomendaciones}

4.5.1. Isquemia miocárdica y arritmias

Dado que ambas pueden ser complicaciones del ictus agudo, se recomienda la monitorización cardíaca durante las primeras fa- ses de evaluación del paciente y la realización de electrocardiogramas (ECG) seriados durante las primeras 48 horas (grado C) $[1,2,42]$. El tratamiento específico debe aplicarse si son persistentes o tienen repercusión clínica.

\subsubsection{Infecciones}

Se recomienda el empleo precoz de antibióticos para las complicaciones infecciosas. Se buscará el foco de infección iniciando antibioterapia empírica y específica tras el antibiograma.

\subsubsection{Trombosis venosa profunda y tromboembolismo pulmonar}

Se recomienda el uso de heparinas de bajo peso molecular, heparinoides o aspirina para la prevención de trombosis venosa profunda y embolismo pulmonar en pacientes inmovilizados y con parálisis o paresia grave de los miembros inferiores (grado A) [43-48].

En pacientes con contraindicación para la medicación antitrombótica se deben utilizar medias de compresión intermitente (grado B) [49].

$\mathrm{Si}$ se produce una trombosis venosa profunda o un tromboembolismo pulmonar el tratamiento debe ser anticoagulación con heparina no fraccionada intravenosa (en dosis para mantener 1,5-2 veces el control de TTPa) seguida de anticoagulantes orales (INR 2,5-3) o bien heparinas de bajo peso en dosis anticoagulantes según el peso del paciente.

\subsection{Tratamiento específico de la isquemia cerebral en fase aguda}

4.6.1. Medidas dirigidas a mejorar

$o$ restablecer el FSC. Recomendaciones.

- Antitrombóticos. Se recomienda iniciar el tratamiento con aspirina precozmente, dentro de las primeras 48 horas tras un infarto cerebral salvo contraindicación (grado A, niveles de evidencia I y II) [1,2,46-48].

Las heparinas no fraccionadas de bajo peso molecular o los heparinoides no ofrecen beneficio neto sobre los antiagregantes en cuanto a reducción de recurrencias precoces o mejoría de la evolución cuando se utilizan en los primeros 14 días tras un infarto cerebral, por lo que no se recomiendan con otro objeto que la prevención de la trombosis venosa (evidencia I y II, grado A) [1,2,45,50-55].

Si se realiza trombólisis está contraindicado usar cualquier antitrombótico en las primeras 24 horas tras el tratamiento con rt-PA (grado A). Se recomienda no usar ningún antitrombótico hasta descartar la indicación de trombólisis [1,2].

Hay acuerdo en recomendar el tratamiento del infarto cerebral agudo de origen cardioembólico considerado de alto riesgo de recidivas precoces con heparina IV, salvo contraindicación (grado C) [1,2]. El tratamiento irá seguido de anticoagulación oral para la prevención secundaria a largo plazo. Aunque no hay datos suficientes para recomendar el uso de heparina IV en infartos progresivos o AIT de repetición a pesar del tratamiento, su empleo es una práctica clínica que se acepta, salvo contraindicación (grado C).

No está establecida la utilidad de la anticoagulación urgente en pacientes con infartos del territorio vertebrobasilar ni en pacientes con disección arterial.

- Trombolíticos. Se recomienda el tratamiento trombolítico con rtPA intravenoso en dosis de $0,9 \mathrm{mg} / \mathrm{kg}$ para el tratamiento del infarto cerebral agudo en el territorio carotídeo, de menos de tres horas de evolución (nivel I, grado A) [56-62]. 
Tabla VIII. Tratamiento trombolítico con rtPA.

Criterios de inclusión

Pacientes con ictus isquémico agudo de menos de tres horas de evolución en los que no concurra alguno de los siguientes criterios de exclusión

Criterios de exclusión

\section{Edad > 80 años}

Hemorragia intracraneal en la TAC

Evolución de los síntomas superior a tres horas o desconocimiento de la hora de inicio

Síntomas menores o en mejoría franca antes del inicio de la infusión Ictus grave según criterios clínicos (NIHSS > 25) o de neuroimagen

Crisis comiciales al inicio del ictus

Síntomas sugestivos de hemorragia subaracnoidea aunque la TAC sea normal

Tratamiento con heparina en las 48 horas previas y TTPa elevado

Ictus previo en cualquier momento e historia concomitante de diabetes

Ictus en los tres meses previos

Plaquetas por debajo de $100.000 / \mathrm{mm}^{3}$

Glucemia por debajo de $50 \mathrm{mg} / \mathrm{dL}$ o por encima de $400 \mathrm{mg} / \mathrm{dL}$

Presión arterial sistólica superior a $185 \mathrm{mmHg}$, presión arterial diastólica superior a $110 \mathrm{mmHg}$ o necesidad de medidas agresivas para bajar la tensión arterial a estos límites

Diatesis hemorrágica conocida

Tratamiento con anticoagulantes orales

Sangrado grave reciente o manifiesto

Historia de hemorragia intracraneal

Antecedentes de HSA por rotura aneurismática

Historia de lesión del sistema nervioso central (aneurismas neoplasias, cirugía intracraneal o medular)

Retinopatía hemorrágica (p. ej., retinopatía diabética)

Antecedentes de masaje cardíaco, parto o punción

en un vaso sanguíneo no accesible en los 10 días previos

Endocarditis bacteriana, pericarditis

Pancreatitis aguda

Enfermedad ulcerativa gastrointestinal documentada en los tres meses previos, varices esofágicas, malformaciones vasculares intestinales conocidas

Neoplasia con aumento del riesgo de sangrado

Enfermedad hepática grave (insuficiencia hepática, cirrosis, hipertensión portal, hepatitis activa)

Cirugía mayor o traumatismo significativo en los tres meses previos

Pauta de administración de rtPA

Se administrarán 0,9 mg/kg; la dosis máxima es de $90 \mathrm{mg}$

El $10 \%$ de la dosis total se administra en bolo durante un minuto

El resto de la dosis se administra en infusión continua durante una hora
Recomendaciones sobre el manejo general

y tratamientos concomitantes

No se administrará heparina o anticoagulantes orales en las siguientes 24 horas, porque pueden aumentar el riesgo de hemorragia cerebral

Se debe ser cauto en pacientes en tratamiento antiagregante previo, ya que esto puede aumentar el riesgo de hemorragia cerebral, aunque éste se correlaciona más con el tamaño y la gravedad del infarto

El paciente debe monitorizarse, preferiblemente en una unidad deictus

Se realizará una exploración neurológica cada 15 minutos durante la infusión, cada 30 minutos durante las siguientes 6 horas y cada hora en las siguientes 24 horas

La infusión debe interrumpirse si hay sospecha clínica de sangrado (cefalea intensa, vómitos, disminución del nivel de conciencia, empeoramiento del déficit) y se realizará TAC craneal urgente

Evitar en lo posible o retrasar al máximo la colocación de sondas urinarias o nasogástricas y punciones arteriales

En el caso de sobredosificación suele producirse consumo de fibrinógeno y otros factores de coagulación. Generalmente es suficiente con esperar la regeneración fisiológica de estos factores; si se produce hemorragia, seguir las recomendaciones para este caso

Control de la TA

LaTA debe ser inferior a 185/110 $\mathrm{mmHg}$ antes de iniciar la infusión

Si la TA supera 185/110 en dos determinaciones separadas 5-10 minutos, administrar uno o dos bolos de 10-20 mg de labetalol IV separados 20 minutos o un parche de nitroglicerina subcutánea. Si con estas medidas no baja la TA, no debe administrarse trombólisis. Si se produce la elevación una vez iniciada la infusión y no se consigue bajar, se debe interrumpir la infusión

Si se produce una reacción anafiláctica (raro) suspender la infusión e iniciar las medidas oportunas

Se monitorizará la tensión arterial cada 15 minutos durante la infusión y la primera hora tras la misma, cada 30 minutos durante las siguientes 6 horas y cada hora hasta completar 24 horas. La frecuencia de los controles debe ser mayor si la TA llega a 180/105 mmHg

Tratamiento de la hemorragia tras la trombólisis

Debe sospecharse de hemorragia cerebral cuando aparece deterioro neurológico, cefalea intensa, vómitos o elevación aguda de la TA

Puede producirse hemorragia sistémica visible u oculta (alteración hemodinámica)

Detener la infusión de rtPA

Realizar TAC craneal urgente (para la hemorragia cerebral)

Determinar tiempos de coagulación, fibrinógeno, recuento plaquetario y realizar pruebas cruzadas

Valorar transfusión de crioprecipitado rico en factor VIII y plaquetas, plasma fresco o sangre fresca

Si persiste la hemorragia, plantear el uso de antifibrinolíticos (ácido tranexámico o aprotinina) 
Tabla IX. Manejo de la tensión arterial en la hemorragia cerebral.

Fármacos de elección

Labetalol: 20 mg en 1-2 minutos. Repetir cada 10-20 minutos hasta el control de la TA o una dosis máxima de 200-300 mg; se mantiene con dosis cada 6-8 horas, según las necesidades

Enalapril: un bolo de $1 \mathrm{mg}$. Repetir cada seis horas 1-5 mg según las cifras de TA

Nitroprusiato sódico: $2 \mathrm{mg} / \mathrm{kg} / \mathrm{min}$

Hipertensión arterial

Si la PAS es mayor de $230 \mathrm{mmHg}$ o la PAD es mayor de $140 \mathrm{mmHg}$ en dos mediciones separadas por más de cinco minutos, iniciar nitroprusiato

Si la PAS es 180-230, la PAD es 105-140 o la presión arterial media (PAM) $130 \mathrm{mmHg}$ o más en dos mediciones separada más de 20 minutos, iniciar labetalol, enalapril u otro antihipertensivo en dosis bajas (tales como diltiacem, lisinopril o verapamil)

Si la PAS es menor de $180 \mathrm{mmHg}$ y la PAD es menor de $105 \mathrm{mmHg}$, diferir el tratamiento antihipertensivo

Si la presión intracraneal está monitorizada, la presión de perfusión cerebral debe mantenerse en valores superiores a $70 \mathrm{mmHg}$

Hipotensión arterial

La primera actitud terapéutica debe centrarse en reponer líquidos. Puede utilizarse suero fisiológico o expansores de plasma, vigilando la presión venosa central. Si la hipotensión persiste tras corregir

el déficit de volumen, deben considerarse los fármacos vasopresores, especialmente indicados si la PAS es menor de $90 \mathrm{mmHg}$

Los fármacos que se han de usar son: fenilefrina $(2-10 \mu \mathrm{g} / \mathrm{kg} / \mathrm{min})$ dopamina (2-20 $\mu \mathrm{g} / \mathrm{kg} / \mathrm{min}$ ) y norepinefrina (desde 0,05-0,2 $\mu \mathrm{g} / \mathrm{kg} / \mathrm{min}$ )

La selección debe ser cuidadosa, siguiendo estrictamente los criterios establecidos.

En Europa se recomienda el uso del rtPA siguiendo el protocolo del registro Safety Implementation of Thrombolysis in Stroke-Monitoring Study (SITS-MOST) [63]. Las normas de administración, así como el tratamiento de la hipertensión arterial o de las complicaciones hemorrágicas se indican en la tabla VIII.

El tratamiento trombolítico sólo lo debe indicar y administrar un neurólogo experto entrenado en el manejo del paciente con ictus y en la interpretación de la TAC craneal, y sólo se administrará en centros con medios suficientes para el cuidado específico de estos pacientes -preferiblemente en una unidad de ictus-, así como para el tratamiento de las posibles complicaciones.

En los pacientes tratados con trombólisis intravenosa no se deben utilizar antiagregantes o anticoagulantes en las 24 horas siguientes.

Ningún otro agente trombolítico intravenoso ha demostrado un perfil de eficacia y seguridad superponible al de rtPA, por lo que no deben usarse en el tratamiento del infarto cerebral agudo [64-71].

Por el momento, sólo se puede recomendar la trombólisis intraarterial en centros seleccionados de manera protocolizada, y nunca debe sustituir a la trombólisis con rt-PA intravenosa cuando ésta se indica [73-75].

\subsubsection{Neuroprotectores}

A pesar de los resultados prometedores de los estudios experi- mentales, muy pocos fármacos los han reproducido en ensayos clínicos. Un ejemplo de éstos es la citicolina, que ha mostrado eficacia en estudios aleatorizados, con el beneficio añadido de su escasa toxicidad. Próximamente un nuevo estudio en fase III ofrecerá mayores evidencias sobre la utilidad de la citicolina en el infarto cerebral agudo [76-81]. Hasta ahora, no hay datos suficientes para recomendar el uso rutinario de neuroprotectores en el tratamiento del infarto cerebral fuera del ensayo clínico. Los fármacos que hayan probado su seguridad y demostrado algún grado de eficacia (p. ej., la citicolina) podrían usarse de forma estrictamente protocolizada [76-82].

\section{TRATAMIENTO DE LA TROMBOSIS VENOSA CEREBRAL. RECOMENDACIONES}

Se recomienda el tratamiento con heparina no fraccionada como primera elección o, en su defecto, heparina de bajo peso molecular durante la fase aguda de la trombosis de senos durales, incluso en presencia de infarto hemorrágico, seguido de anticoagulación oral (INR 2,0-3,0) durante 3-6 meses (evidencia I y recomendación A) [1-5].

\section{ACTUACIÓN CLÍNICA EN LA HEMORRAGIA CEREBRAL (HC) \\ 6. 1. Valoración inicial y diagnóstico etiológico. Recomendaciones}

La TAC craneal es la técnica de neuroimagen de elección en la valoración inicial de un paciente en el que se sospeche HC [1,2].

La resonancia magnética cerebral con angiografía (ARM) está indicada en pacientes jóvenes para descartar cavernomas, en pacientes normotensos con arteriografía normal y HC lobares, sobre todo si son susceptibles de cirugía, y como método de cribado en pacientes ancianos no hipertensos con hemorragias lobares. La detección de microhemorragias múltiples por RM puede modificar el control y tratamiento de la HTA y el pronóstico de los pacientes. Las nuevas técnicas de RM multimodal y la mayor disponibilidad de equipos en los hospitales permite que se considere de primera elección en pacientes sin factores de riesgo.

La angiografía por RM permite el diagnóstico de malformaciones vasculares y aneurismas en muchos casos [3-5].

La arteriografía debe valorarse en todos los pacientes con HC de etiología no aclarada que puedan ser candidatos a cirugía, sobre todo si son jóvenes y están clínicamente estables. El momento de realización de la arteriografía depende del estado clínico del paciente y de la urgencia del acto quirúrgico [6,7].

La angiografía no es necesaria en pacientes hipertensos mayores de 45 años si en la TAC no hay signos sugerentes de malformación vascular o de lesión estructural $[5,7]$.

\subsection{Tratamiento en la fase aguda}

\subsubsection{Cuidados generales. Recomendaciones}

Se recomienda la atención de todo paciente con HC en hospitales dotados con los medios adecuados para el correcto diagnóstico y tratamiento. Se recomienda la atención por un neurólogo, preferentemente en unidades de ictus, salvo que requieran cirugía o ventilación asistida, en cuyo caso deben ingresar en la UCI $[2,5,8]$.

Se recomienda un adecuado control de la presión arterial con reducción de la presión arterial sistólica hasta 190/110 en las seis primeras horas. Se recomienda iniciar el tratamiento 
con labetalol intravenoso o enalapril (por vía oral o intravenosa) si la PAS es de 180230, la PAD 105-140 o la presión arterial media es de 130 o superior. En casos de PAS mayor de 230 o PAD mayor de 140 se recomienda el tratamiento con nitroprusiato. En casos de hipotensión se recomienda utilizar suero fisiológico o expansores de plasma y en casos resistentes se podrían emplear fármacos vasopresores [9-11] (Tabla IX).

Se recomienda utilizar medias compresivas para la prevención de trombosis venosa profunda y embolismo pulmonar.

\subsubsection{Tratamiento del aumento \\ de la presión intracraneal (PIC). \\ Recomendaciones}

Se recomienda evitar y tratar todos aquellos factores que pueden contribuir a incrementar la PIC (hipoxemia, hipercarbia, hipertermia, convulsiones y todas aquellas condiciones que aumenten la presión intratorácica) [12-14].

Se recomienda la utilización de agentes osmóticos como primera elección, pero no deben aplicarse de forma profiláctica. El manitol al $20 \%(0,25-0,5 \mathrm{~g} / \mathrm{kg} / 4 \mathrm{~h})$ se inicia en pacientes con ondas tipo B, valores de PIC que aumentan progresivamente o signos clínicos de deterioro asociados con efecto de masa. Para evitar el efecto de rebote es conveniente no utilizarlo más de cinco días. La furosemida (10 $\mathrm{mg} / 2-8 \mathrm{~h}$ ) puede utilizarse de forma simultánea para mantener el gradiente osmótico. Es necesario medir la osmolaridad del plasma dos veces al día, y debe mantener valores de $310 \mathrm{mOsm} / \mathrm{L}$ [15] o menores.

Se deben evitar los corticoides [16].

Se recomienda hiperventilación si fallan los agentes osmóticos. La reducción de la $\mathrm{pCO}_{2}$ hasta 25-30 mmHg consigue una disminución de la PIC del 25-30\% en la mayoría de los pacientes. La ausencia de respuesta indica mal pronóstico. Su máxima utilidad se obtiene en las primeras 48 horas y no deberá usarse más de 4-5 días para evitar efecto de rebote [17].

La utilidad de monitorizar la PIC no está bien establecida [18].

Se recomienda el tratamiento con benzodiacepinas de vida media corta o neurolépticos y analgésicos en caso de agitación.

\subsubsection{Tratamiento quirúrgico. Recomendaciones}

No se recomienda la cirugía en los siguientes casos:

- Pacientes con HC de volumen menor de $10 \mathrm{~mL}$ o con déficit neurológico mínimo [19-23].

- Pacientes con puntuación en la escala de Glasgow de 3 o 4. Si la hemorragia es cerebelosa, incluso con estas puntuaciones puede valorarse la cirugía [24,25].

- Hemorragias profundas [26].

Se recomienda cirugía en los siguientes casos:

- Hemorragia cerebelosa mayor a $3 \mathrm{~cm}$, con deterioro neurológico, compresión de tronco o hidrocefalia. Debe operarse de forma urgente $[2,5,27,28]$.
- HC asociada a lesión estructural (aneurisma, malformación arteriovenosa o angioma cavernoso), que tiene posibilidad de un buen pronóstico funcional, y cuya lesión estructural es accesible a la cirugía.

- En pacientes jóvenes con hemorragia lobar de tamaño moderado o grande, sin sospecha de angiopatía amiloide, que cursan con deterioro neurológico [2,5,21-23].

\subsection{Prevención secundaria de la $\mathrm{HC}$.}

\section{Tratamiento según etiología. Recomendaciones}

Se recomienda tratamiento de la HTA y control estricto procurando la normotensión, manteniendo cifras por debajo de 140/ $80 \mathrm{mmHg}$ [29-31].

En caso de cavernomas, se recomienda la cirugía en las lesiones accesibles, y en las profundas, un estrecho seguimiento con indicación de la cirugía en casos seleccionados [32,33].

En las malformaciones arteriovenosas se recomienda el tratamiento quirúrgico, endovascular o la radiocirugía, o bien la combinación de varios, en función de su localización, forma y tamaño [34,35].

En las HC secundarias a tumores se aconseja el tratamiento quirúrgico del tumor subyacente, siempre que el tumor sea accesible, que la exéresis sea curativa (p. ej., tumores benignos) o mejore la calidad de vida o la supervivencia y el riesgo quirúrgico no sea mayor que el beneficio.

En las HC por vasculitis, inflamatorias o secundarias a fármacos podrían emplearse corticoides.

En la HC secundaria a terapia anticoagulante o trombolítico 
Tabla Xa. Escalas de valoración en la HSA (Hunt y Hess).

\begin{tabular}{|c|c|}
\hline Grado 0 & Aneurisma intacto \\
\hline Grado 1 & Asintomático o mínima cefalea; rigidez de nuca ligera \\
\hline Grado $1 \mathrm{~A}$ & $\begin{array}{l}\text { Sin reacción meníngea o cerebral pero con déficit } \\
\text { neurológico establecido }\end{array}$ \\
\hline Grado 2 & $\begin{array}{l}\text { Cefalea moderada o grave; rigidez de nuca; sin déficit } \\
\text { neurológicos graves a excepción de la afectación de pares } \\
\text { craneales }\end{array}$ \\
\hline Grado 3 & Somnolencia, confusión o déficit focales leves \\
\hline Grado 4 & $\begin{array}{l}\text { Estupor; hemiparesia moderada o grave; alteraciones } \\
\text { vegetativas y posiblemente rigidez de descerebración precoz. }\end{array}$ \\
\hline o 5 & erebración. \\
\hline
\end{tabular}

Tabla Xc. Escalas de valoración en la HSA (escala World Federation of Neurological Surgeons, WFNS)

\begin{tabular}{llll}
\hline & Parámetros & & Resultado \\
\cline { 1 - 1 } Glasgow & Déficit motor & Grado WFNS \\
\hline 15 & Ausente & I & II \\
\hline $14-13$ & Ausente & III \\
\hline $14-13$ & Presente & IV \\
\hline $12-7$ & - & $\mathrm{V}$
\end{tabular}

se recomienda suspender el tratamiento, iniciar transfusión de plasma fresco o crioprecipitados y vitamina $\mathrm{K}$ de forma inmediata en los casos de tratamiento anticoagulante. Se podría recomendar la cirugía en casos seleccionados, una vez revertido el efecto anticoagulante o trombolítico [36].

En la HC asociada a trombopenia debe realizarse la transfusión de plaquetas. En pacientes con trombopenia autoinmune se recomiendan corticoides, especialmente si se va a realizar cirugía.

\section{MANEJO CLÍNICO DE LA}

\section{HEMORRAGIA SUBARACNOIDEA}

\section{1. Sistemática diagnóstica. Recomendaciones (Fig. 2)}

La sistemática diagnóstica se recoge en la figura 2.

Ante la sospecha clínica de HSA, se debe referir inmediatamente a un centro especializado para su tratamiento.

Se recomienda realizar una TAC cerebral ante la sospecha de HSA, al igual que una punción lumbar en el caso que la TAC sea negativa. La RM que se realiza con algunas secuencias de exploración (eco de gradiente $\mathrm{T}_{2}$, FLAIR, combinaciones de secuencias) puede ser una alternativa [1-4].

La angiografía cerebral selectiva es imprescindible en el diagnóstico de la HSA. En el caso de estar contraindicada, se puede sustituir por una angiorresonancia magnética (ARM) o por una TAC helicoidal con contraste (grado C) [5].

Se recomienda realizar una segunda angiografía en aquellos casos de HSA no perimesencefálica con una primera angiografía negativa, después de transcurrir al menos dos semanas (grado C) [6].

El Doppler transcraneal (DTC) se recomienda para el diagnóstico y monitorización del vasoespasmo (grado C) [7].
Tabla Xb. Escala de Glasgow

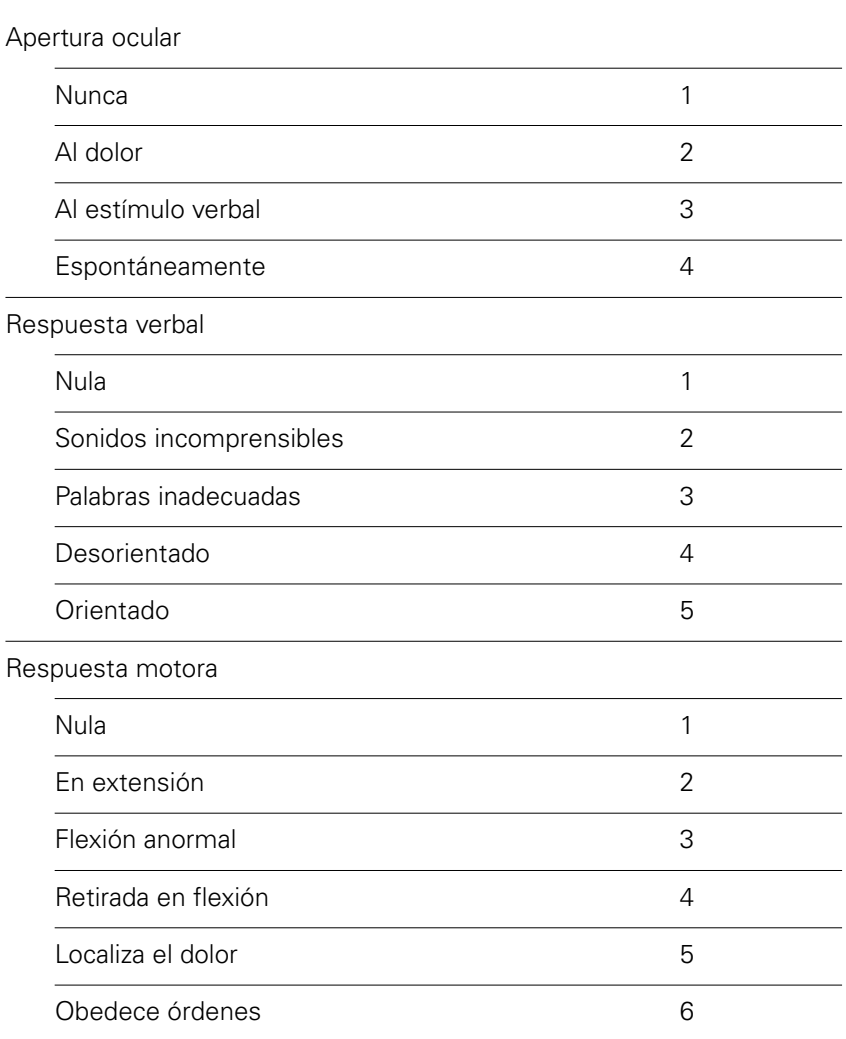

\subsection{Manejo de los pacientes con aneurismas} cerebrales que no se han roto. Recomendaciones

La ARM constituye una buena prueba para la detección de los aneurismas no rotos [8].

Las personas con más de un familiar directo con aneurismas íntegros o HSA aneurismática y los pacientes que ya se han tratado mediante HSA por ruptura de un aneurisma son las subpoblaciones en las que pudiera estar indicada la práctica rutinaria de estos programas de detección de aneurismas mediante ARM (grado C) [8,9].

El tratamiento quirúrgico o endovascular de los aneurismas no rotos debe considerarse de forma individualizada [10-12].

Se puede recomendar el tratamiento quirúrgico o endovascular de los aneurismas no rotos asintomáticos en pacientes jóvenes (menos de 45 años), sin enfermedades concomitantes, con un tamaño mayor de $10 \mathrm{~mm}$, localizados en el ápex de la arteria basilar, con saco aneurismático lobulado y con historia personal o familiar previa de HSA (grado C) $[8,13,14]$.

Se considerará también el tratamiento quirúrgico o endovascular en los aneurismas intradurales o intracavernosos de gran tamaño que ejercen efecto compresivo sobre estructuras vecinas (grado C) [14].

No existe evidencia que justifique la realización rutinaria de ARM en pacientes con síndromes genéticos asociados a un alto riesgo de aneurismas cerebrales o pacientes con síndrome de Marfan o de Ehlers-Danlos) [15].

\subsection{Tratamiento médico de la HSA. Recomendaciones}

Están dirigidas a evitar las complicaciones de la HSA: resangrado, vasoespasmo, edema cerebral, hidrocefalia y crisis comiciales.

Se recomienda la monitorización mediante escalas (Tabla X). 
El reposo en cama o el tratamiento antihipertensivo son útiles como medidas coadyuvantes (grado B) [16,17].

El uso de antifibrinolíticos no está indicado (grado A) [18-23]

La terapia triple $\mathrm{H}$ es útil para prevenir el vasoespasmo y debe aplicarse una vez excluido el aneurisma (grado C) [24-29].

Se recomiendan los antagonistas del calcio, nimodipino o nicardipino para la prevención del vasoespasmo. Debe monitorizarse la tensión arterial. El tratamiento se prolongará tres semanas. Si es posible se usará la vía oral (360 mg/día de nimodipino: $60 \mathrm{mg} / 4 \mathrm{~h})$ y si no, la vía intravenosa $(3 \mu \mathrm{g} / \mathrm{kg} / \mathrm{h}$ de nimodipino en infusión continua $\mathrm{y}$, según las cifras de tensión arterial, aumentar la dosis a ritmo de $2 \mathrm{mg} / \mathrm{h}=1 \mathrm{~mL} / \mathrm{h}$ hasta alcanzar la dosis de $30 \mu \mathrm{g} / \mathrm{kg} / \mathrm{h}$ ) durante 14 días, y pasar entonces a administración oral (grado A) [30-37].

La angioplastia transluminal puede ser eficaz en el tratamiento del vasoespasmo resistente a la terapia convencional (grado C) [38,39].

\subsection{Tratamiento de las complicaciones médicas asociadas a la HSA. Recomendaciones}

Se recomienda la implantación de un drenaje de derivación ventriculoperitoneal en la hidrocefalia secundaria a HSA en pa- cientes con deterioro clínico evidente y estudio de neuroimagen compatible (grado C) [40,41].

No existen datos concluyentes que permitan recomendar el uso profiláctico de anticomiciales en la HSA aneurismática (grado C) $[42,43]$.

\subsection{Terapia endovascular o quirúrgica en la hemorragia ubaracnoidea. Recomendaciones}

El objetivo fundamental es la exclusión del aneurisma para evitar el resangrado [44].

Se recomienda la exclusión del aneurisma por técnica endovascular o quirúrgica [45-48].

La terapia endovascular puede ser de primera elección en centros con suficiente experiencia (grado C) [48-52].

La actuación precoz (menos de 72 horas) disminuye el riesgo de resangrado (grado B) [53-57]. No está indicada la cirugía precoz en los pacientes con situación clínica desfavorable (grados de Hunt y Hess IV y V -Tabla X-) (grado B) [48,58-63].

Cuando se ha realizado tratamiento endovascular, es necesario un seguimiento angiográfico al menos durante dos años, para excluir la recanalización parcial del aneurisma embolizado, factor asociado con el resangrado (grado C) [48,64-66].

\section{BIBLIOGRAFÍA}

\section{INTRODUCCIÓN}

1. Barba R, Martínez-Espinosa S, Rodríguez-García E, Pondal M, Vivancos J, Del Ser T. Poststroke dementia: clinical features and risk factors. Stroke 2000; 31: 1494-501.

2. Wolfe C. The impact of stroke. Br Med Bull 2000; 56: 275-86

3. Madureira S, Guerreiro M, Ferro JM. Dementia and cognitive impairment three months after stroke. Eur J Neurol 2001; 8: 621-7.

4. González M, De La Hoz F. Mortalidad por enfermedades cardiovasculares. Colombia 1990-1999. Informe Quincenal Epidemiológico Nacional 2002; 22417-43.

5. Instituto Nacional de Estadística. Defunciones según la causa de muerte 2000. http://www.ine.es. Fecha última consulta: 30.12.2002.

6. Saposnik G, Del Brutto OH. Stroke in South America. A systematic review of incidence, prevalence and stroke subtypes. Stroke 2003; 34: 2103.

7. López-Pousa S, Vilalta J, Llinás J. Incidencia de la enfermedad vascular cerebral en España. Estudio en un área rural en Girona. Rev Neurol 1995; 23: 1074-80.

8. Caicoya M, Rodríguez T, Laceras C, Cuello R, Corrales C, Blázquez B. Incidencia del accidente cerebrovascular en Asturias 1990-1991. Rev Neurol 1996; 24: 806-11.

9. Pérez-Sempere A, Duarte J, Cabezas C, Clavería E. Incidence of transient ischemic attacks and minor stroke in Segovia, Spain. Stroke 1996; 27: 667-71.

10. Bermejo F, Gabriel R, Morales JM. Stroke and TIS in old people in four districts of Madrid, Spain: data from a population based study. Neuroepidemiology 1993; 12: 121.

11. Dávalos A, Castillo J, Martínez Vila E for the Cerebrovascular Study Group of the Spanish Society of Neurology. Delay in neurological attention and stroke outcome. Stroke 1995; 26: 2233-7.

12. Fuentes B, Díez Tejedor E, Lara M, Frank A, Barreiro P. Organización asistencial en el cuidado del ictus. Las Unidades de Ictus marcan la diferencia. Rev Neurol 2001; 32: 101-6.

13. Aboderin J, Venables G. Stroke management in Europe. Pan European Consensus Meeting on stroke management. J Intern Med 1996; 240: 173-80.

14. Sackett DL. Rules of evidence and clinical recommendations on the use of antithrombotic agents. Chest 1986; 89: 2S-3S.

\section{PREVENCIÓN DE LA ISQUEMIA CEREBRAL}

1. Díez-Tejedor E. Infarto cerebral (introducción). Rev Clin Esp 1996; 196 (Suppl 3): S1-2.

2. Sacco RL, Benjamín EJ, Broderick JP, Dyken M, Easton JD, Feinberg WM, et al. Risk factors. AHA Conference Proceedings. Stroke 1997; 28: $1507-17$
3. Gorelick PB, Sacco RL, Smith DB, Alberts MD, Mustone-Alexander $\mathrm{L}$, Rader D, et al. Prevention of a first stroke. A review of guidelines and a multidisciplinary consensus statement from the National Stroke Association. JAMA 1999; 281: 1112-20.

4. Goldstein LB, Adams R, Becker K, Furberg CD, Gorelick PB, Hademenos G, et al. Primary prevention of ischemic stroke. A statement for healthcare professionals from the Stroke Council of the American Heart Association. Stroke 2001; 103: 163-82.

5. European Stroke Iniciative (EUSI) on behalf of the European Stroke Council (ESC), the European Neurological Society (ENS) and the European Federation of Neurological Societies (EFNS). Recommendations for stroke management. Cerebrovasc Dis 2000; 10 (Suppl 3): 1-34.

6. Vasan RS, Larson MG, Leip EP, Evans JC, O'Donnell CJ, Kannel WB, et al. Impact of high-normal blood pressure on the risk of cardiovascular disease. N Engl J Med 2001; 345: 2191-7.

7. Collins R, Peto P, Macmahon S, Herbert P, Fiebach N, Eberlein K. Blood pressure, stroke, and coronary heart disease. 2. Short term reductions in blood pressure: Overview of randomised drug trials in their epidemiological context. Lancet 1990; 335: 827-38.

8. The Heart Outcomes Prevention Evaluation Study Investigators. Effects of an angiotensin-converting enzyme inhibitor, ramipriol, on cardiovascular events in high-risk patients. N Engl J Med 2000; 342: 145-53.

9. UK Prospective Diabetes Study (UKPDS) Group. Intensive blood glucose control with sulphonylureas or insulin compared with conventional treatment and risk complications in patients with type 2 diabetes (UKPDS 33). Lancet 1998; 352: 837-53.

10. UK Prospective Diabetes Study Group. Tight blood pressure control and risk of macrovascular and microvascular complications in type 2 diabetes. UKPDS 38. BMJ 1998; 317: 703-13.

11. Sacks FM, Pfeffer MA, Moye LA, Rouleau JL, Rutherford JD, Cole TG, et al. The effect of pravastatin on coronary events after myocardial infarction in patients with average cholesterol levels. Cholesterol and Recurrent Events Trial Investigators. N Engl J Med 1996; 335: 1001-9.

12. Gil-Núñez AC, Villanueva JA. Advantages of lipid-lowering therapy in cerebral ischemia. Role of HMG-CoA reductase inhibitors. Cerebrovasc Dis 2001; 11 (Suppl 1): 85-95.

13. Díez-Tejedor E, Egido-Herrero JA, Gil-Núñez AC, González-Juanatey JR, López-Pastor A, Marta-Moreno J, et al. ¿Están las estatinas indicadas en la prevención del infarto cerebral? Rev Neurol 2000; 30: 671-93.

14. Byington RP, Davis BR, Plehn JF, White HD, Baker J, Cobbe SM, et al, for PPP Investigators. Reduction of stroke with pravastatin. The Prospective Pravastatin Pooling (PPP) Project. Circulation 2001; 103: 387-392.

15. Di Mascio R, Marchioli R, Tognoni G. Cholesterol reduction and stroke occurrence: an overview of randomized clinical trials. Cerebrovasc Dis 2000; 10: 85-92. 
16. Expert Panel on Detection, Evaluation, and Treatment of High Blood Cholesterol in Adults: Executive Summary of the Third Report of the National Cholesterol Education Program (NCEP) Expert Panel on Detection, Evaluation, and Treatment of High Blood Cholesterol in Adults (Adult Treatment Panel III). JAMA 2001; 285: 2486-97.

17. Wolf PA, D'Agostino RB, Kannel WB, Bonita R, Belanger AJ. Cigarette smoking as a risk factor for stroke: the Framingham Study. JAMA 1988; 259: 1025-9.

18. Peto R, Gray R, Collins R, Wheatley K, Hennekens C, Jamrozik K, et al. Randomized trial of prophylactic daily aspirin in British male doctors. Br Med J 1988; 296: 313-6.

19. Steering Committee of the Physicians' Health Study Research Group. Final report of the ongoing physicians health study. N Engl J Med 1989; 321: 129-35

20. Hayden M, Pignone M, Phillips C, Mulrow C. Aspirin for primary prevention of cardiovacular events: a summary of the evidence for the U.S. Preventive Services. Task Force. Ann Intern Med 2002; 136: 161-72.

21. Hart RG, Halperin JL, McBride R, Benavente O, Man-Son-Hing M, Kronmal RA. Aspirin for the primary prevention of stroke and other major vascular events: meta-analysis and hypotheses. Arch Neurol 2000; 57: 326-32.

22. Díez-Tejedor E, Fuentes B, Gil-Núñez A, Gil-Peralta A, Matías-Guiu J. Guía para el tratamiento preventivo de la isquemia cerebral. Guía para el tratamiento y prevención del ictus. Neurología 2002; 17 (Suppl 3): S61-75.

23. Heras M, Fernández-Ortiz A, Gómez-Guindal JA, Iriarte JA, Lidón RM, Pérez-Gómez F, et al. Guías de actuación clínica de la Sociedad Española de Cardiología. Recomendaciones para el uso del tratamiento antitrombótico en cardiología. Rev Esp Cardiol 1999; 52: 801-20.

24. The BAATAF Investigators. The effect of low-dose warfarin on the risk of stroke in patients with nonrheumatic atrial fibrillation. N Engl J Med 1990; 323: 1505-11.

25. SPAF Investigators. Warfarin versus aspirin for prevention of thromboembolism in atrial fibrillation: Stroke Prevention in Atrial Fibrillation II Study. Lancet 1994; 343: 687-91.

26. SPAF investigators. Adjusted-dose warfarin versus low-intensity, fixed-dose warfarin plus aspirin for high-risk patients with atrial fibrillation: Stroke prevention in atrial fibrillation III randomised clinical trial. Lancet 1994; 348: 633-8.

27. Petersen P, Boisen G, Godtfredsen J, Anderson ED, Andersen B. Placebo-controlled, randomized trial of warfarin and aspirin for prevention of thromboembolic complications in chronic atrial fibrillation: the Copenhagen AFASAK study. Lancet 1989; 1: 175-9.

28. Ezekowitz MD, Bridgers SL, James KE, Carliner NH, Colling CL, Gornick CC, et al, for the Veterans Affairs Stroke Prevention in Nonrheumatic Atrial Fibrillation Investigators. Warfarin in the prevention of stroke associated with non-rheumatic atrial fibrillation. N Engl J Med 1992; 327: 1406-12.

29. Connolly SJ, Laupacis A, Gent M, Roberts RS, Cairns JA, Joyner C. Canadian Atrial Fibrillation Anticoagulation (CAFA) Study. J Am Coll Cardiol 1991; 18: 349-55.

30. The Atrial Fibrillation Investigators. The efficacy of aspirin in patients with atrial fibrillation: analysis of pooled data from 3 randomized trials. Arch Intern Med 1997; 157: 1237-40.

31. Kalral-Pérez I, Melbourn A. Risk assessment and anticoagulation for primary stroke prevention in atrial fibrilation. Stroke 1999; 30: 314-23.

32. The CASANOVA Study Group. Carotid surgery versus medical therapy in asymptomatic carotid stenosis. Stroke 1991; 22: 1229-35.

33. Mayo Asymptomatic Carotid Endarterectomy Study Group. Results of a randomized controlled trial of carotid endarterectomy for asymptomatic carotid stenosis. Mayo Clin Proc 1992; 67: 513-8.

34. Hosbon RW, Weiss G, Fields WS, Goldstone J, Moore WS, Towne JB, et al and the Veterans Affairs Cooperative Study Group. Efficacy of carotid endarterectomy for asymptomatic carotid stenosis. N Engl Med J 1993; 328: 221-27.

35. Goldstein LB, Adams R, Becker K, Furberg CD, Gorelick PB, Hademenos $\mathrm{G}$, et al. Primary prevention of ischemic stroke: a statement for healthcare professionals from the Stroke Council of the American Heart Association. Circulation 2001; 103: 163-82.

36. Biller J, Feinberg WM, Castaldo JE, Whittemore AD, Harbaugh RE, Dempsey RJ, et al. Guidelines for carotid endarterectomy. A statement for healthcare professionals from a special writting group of the Stroke Council, American Heart Association. Stroke 1998; 29: 554-62.

37. Benavente O, Moher D, Pham B. Carotid endarterectomy for asymptomatic carotid stenosis: a meta-analysis. Br Med J 1998; 317: 1477-80.

38. Inzitari D, Eliasziw M, Gates P, Sharpe BL, Chan RKT, Meldrum HE, et al, for The North American Symptomatic Carotid Endarterectomy Trial (NASCET) Group: The causes and risk of stroke in subjects with an asymptomatic internal carotid artery stenosis. N Engl J Med 2000; 342: 1693-700.
39. Halliday AW, Thomas D, Mansfield A. The asymptomatic carotid surgery trial (ACST) rationale and design. Eur J Vasc Surg 1994; 8: 703-10.

40. The ALLHAT officers and coordinators for the ALLHAT Collaborative Research Group. Major outcomes in high-risk hypertensive patients randomized to angiotensin-converting enzyme inhibitor or calcium channel blocker vs diuretic. JAMA 2002; 288: 2981-97.

41. Chobanian AV, Bakris GL, Black HR, Cushman WC, Green LA, Izzo JL Jr, et al. The seventh report of the Joint National Committee on Prevention, Detection, Evaluation, and Treatment of High Blood Pressure: The JNC 7 Report. JAMA 2003; 289: 2560-72.

42. 2003 European Society of Hypertension-European Society of Cardiology guidelines for the management of arterial hypertension. J Hypertens 2003; 21: 1011-53.

43. Douglas JG, Bakris GL, Epstein M, Ferdinand KC, Ferrario C, Flack $\mathrm{JM}$, et al. Management of high blood pressure in African Americans. Consensus Statement of the Hypertension in African Americans Working Group of the International Society on Hypertension in Blacks. Arch Intern Med 2003; 163: 525-41.

44. Staessen JA, Wang J, Thijs L. Cardiovascular prevention and blood pressure reduction: a quantitative overview updated until 1 march 2003 . J Hypertens 2003; 21: 1055-76.

45. Psaty B, Lumley T, Furberg C, Schellenbaum G, Pahor M, Alderman $\mathrm{MH}$, et al. Health outcomes associated with various antihypertensive therapies used as first-line agents. A network meta-analysis. JAMA 2003; 289: 2534-44

46. The Heart Outcomes Prevention Evaluation Study Investigators. Effects of an angiotensin-converting-enzyme inhibitor, ramipril, on death from cardiovascular causes, myocardial, and stroke in high-risk patients. N Engl J Med 200; 342: 145-53.

47. Dahlof B, Devereux RB, Kjeldsen SE, Julius S, Beevers G, De Faire U, et al, for the LIFE study group. Cardiovascular morbidity and mortality in the Losartan Intervention for Endpoint reduction in hypertension study (LIFE): a randomized trial against atenolol. Lancet 2002; 359: 995-1003.

48. PROGRESS Collaborative Group. Effects of blood pressure lowering with perindropil and indapamide therapy on dementia and cognitive decline in patients with cerebrovascular disease. Arch Intern Med 2003; 163: 1069-75.

49. PROGRESS Collaborative Group. Randomised trial of a perindoprilbased blood-pressure-lowering regimen among 6105 individuals with previous stroke or transient ischaemic attack. Lancet 2001; 358: 1033-41.

50. Friday G, Alter M, Lai SM. Control of hypertension and risk of stroke recurrence. Stroke 2002; 32: 2652-7.

51. Anderson C. Blood pressure-lowering for secondary prevention of stroke: ACE inhibitors is the key. Stroke 2003; 34: 1333-4.

52. Path P. Blood pressure-lowering for secondary prevention of stroke: ACE inhibitors is not the key. Stroke 2003; 34: 1334-5.

53. Davies SM, Donnan GA. Blood pressure-lowering for secondary prevention of stroke: mechanism uncertain. Stroke 2003; 34: 1335-6.

54. Scandanavian Simvastatin Survival Study Group. Randomized trial of cholesterol-lowering in 4,444 patients with coronary heart disease: the Scandanavian Simvastatin Survival Study (4S). Lancet 1994; 344: 1383-9.

55. Long-term Intervention with Pravastatin in Ischemic Disease (LIPID) Study Group. Prevention of cardiovascular events and death with pravastatin in patients with coronary arterial disease and a broad range of initial cholesterol levels. N Engl J Med 1998; 339: 1349-57.

56. Blauw GJ, Lagaay M, Smelt AHM, Westendorp RGJ. Stroke, statins and cholesterol. A meta-analysis of randomized, placebo controlled doubleblind trials with HMG-CoA reductase inhibitors. Stroke 1997; 28 : 946-50.

57. Schwartz GG, Olsson AG, Ezekowitz MD, Ganz P, Oliver MF, Waters D, et al, for the Myocardial Ischemia Reduction with Aggressive Colesterol Lowering (MIRACL) Study Investigators. Effects of atorvastatin on early recurrent ischemic events in acute coronary syndromes. The MIRACL study: a randomized controlled trial. JAMA 2001; 285: 1711-8.

58. Hart RG. Oral anticoagulants for secondary prevention of stroke. Cerebrovasc Dis 1997; 7 (Suppl 6): 24-9.

59. Downs JR, Clearfield M, Weis S, Shapiro DR, Beere PA, Langendorfer A, et al, for the AFCAPS/TexCAPS Research Group. Primary prevention of acute coronary events with lovastatin in men and women with average cholesterol levels. JAMA 1998; 279: 1615-22.

60. Shepherd J, Cobbe SM, Ford I, Isles CG, Lorimer AR, Macfarland PW, et al, for the West Scotland Coronary Prevention Study Group. Prevention of coronary heart disease with pravastatin in men with hypercholesterolemia. N Engl J Med 1995; 333: 1301-7.

61. Antiplatelets Trialists' Collaboration. Collaborative overview of randomised trials of antiplatelet therapy. Prevention of death, myocardial infarction and stroke by prolonged antiplatelet therapy in various categories of patients. Br Med J 1994; 308: 81-106.

62. Algra A, Van Gijn K. Secondary prevention after cerebral ischaemia of 
presumed arterial origin: is aspirin still the touchstone? J Neurol Neurosurg Psychiatry 1999; 66: 557-9.

63. Algra A Van Gijn J. Cumulative meta-analysis of aspirin efficacy after cerebral ischemia of arterial origin. J Neurol Neurosurg Psychiatry 1999; 66: 255

64. The Dutch TIA Trial Study Group. A comparison of two doses of aspirin (30 mg vs $283 \mathrm{mg}$ a day) in patients after a transient ischemic attack or minor stroke. N Engl J Med 1991; 325: 1261-6.

65. UK-TIA Study Group. United Kingdom Transient Ischaemic Attack (UK-TIA) Aspirin Trial: final results. J Neurol Neurosurg Psychiatry 1991; 54: 1044-54.

66. The SALT collaborative Group. Swedish Aspirin Low-dose Trial (SALT) of $75 \mathrm{mg}$ aspirin as secondary prophylaxis after cerebrovascular ischaemic events. Lancet 1991; 338: 1345-9.

67. Hart RG, Harrison MJR. Aspirin wars. The optimal dose of aspirin to prevent stroke. Stroke 1996; 27: 585-7.

68. Hass WK, Easton DJ, Adams HP Jr, Pryse-Phillips W, Molony BA, Anderson $\mathrm{S}$, et al. A randomized trial comparing ticlopidine hydrochloride with aspirin for the prevention of stroke in high risk patients. N Engl J Med 1989; 321: 501-7.

69. Gent M, Blakely JA, Easton JD, Ellis DJ, Hachinski VC, Harbison JW, et al. The Canadian American Ticlopidine Study (CATS) in thromboembolic stroke. Stroke 1988; 19: 1203-10.

70. CAPRIE Steering Committee. A randomised, blinded trial of clopidogrel versus aspirin in patients at risk of ischaemic events (CAPRIE). Lancet 1996; 348: 1329-39.

71. Matías-Guiu J, Ferro JM, Álvarez-Sabin J, Torres F, Jiménez MD, et al, TACIP Investigators. Comparison of triflusal and aspirin for prevention of vascular events in patients after cerebral infarction: the TACIP study. A randomized, double-blind, multicenter trial. Stroke 2003; 34: 840-8.

72. Culebras A, Rotta-Escalante R, Vila J, Domínguez R, Abiusi G, Famulari A, et al. Triflusal vs aspirin for prevention of cerebral infarction. A randomised stroke study. Neurology 2004; 62: 1073-80.

73. Bousser MG, Eschwege E, Haguenau M, Lefaucconnier JM, Thibult $\mathrm{N}$, Touboul D, et al. AICLA controlled trial of aspirin and dipyridamole in the secondary prevention of atherothrombotic cerebral ischemia. Stroke 1983; 14: 5-14.

74. American-Canadian Co-Operative Study Group. Persantine aspirin trial in cerebral ischemia. 2. Endpoint results. Stroke 1985; 16: 406-15.

75. Diener HC, Cunha L, Forbes C, Sivenius VJ, Smets P, Lowenthal A. European Stroke Prevention Study 2. Dipyridamole and acetylsalicilic acid in the secondary prevention of stroke. J Neurol Sci 1996; 143: 1-13.

76. Ringleb PA, Bhatt DL, Hirsch AT, Topol EJ, Hacke W, Clopidogrel Versus Aspirin in Patients at Risk of Ischemic Events Investigators. Benefit of clopidogrel over aspirin is amplified in patients with a history of ischemic events. Stroke 2004; 35: 528-32.

77. The Stroke Prevention in Reversible Ischemia Trial (SPIRIT) Study Group. A randomized trial of anticoagulants versus aspirin after cerebral ischemia of presumed arterial origin. Ann Neurol 1997; 42: 857-65.

78. Mohr JP, Thompson JL, Lazar RM, Levin B, Sacco RL, Furie KL, et al, for the Warfarin-Aspirin Recurrent Stroke Study Group. A comparison of warfarin and aspirin for the prevention of recurrent ischemic stroke. N Engl J Med 2001; 345: 1444-51.

79. Chimowitz MI, Kokkinos J, Strong J, Brown MB, Levine SR, Silliman $\mathrm{S}$. The warfarin-aspirin symptomatic intracraneal disease study. Neurology $1995 ; 45: 1488-93$.

80. North American Symptomatic Carotid Endarterectomy Trial Collaborators. Beneficial effect of carotid endarterectomy in symptomatic patients with high-grade carotid stenosis. N Engl J Med 1991; 325: 445-53.

81. European Carotid Surgery Trialists' Collaborative Group. MRC European Carotid Surgery Trial: interim results for symptomatic patients with severe $(70-99 \%)$ or with mild $(0-29 \%)$ carotid stenosis. Lancet 1991; 337: 1235-43.

82. European Carotid Surgery Trialists' Collaborative Group. Endarterectomy for moderate symptomatic carotid stenosis: interim results from the MRC European Carotid Surgery Trial. Lancet 1996; 347: 1591-3.

83. Delgado-Bona G, Gállego-Culleré J, Gil-Peralta A, González-Marcos JR. Endarterectomía y angioplastia transluminal percutánea carotídea en la prevención del infarto cerebral. Rev Clin Esp 1996; 196: 46-9.

84. Rothwell PM, Warlow CP. Prediction of benefit from carotid endarterectomy in individual patients: a risk modelling study. Lancet 1999; 353: 2105-7.

85. Gil-Peralta A, Mayol A, González-Marcos JR. Percutaneous transluminal angioplasty of the symptomatic atherosclerotic carotid arteries. Stroke 1996; 27: 2271-3.

86. The CAVATAS investigators. Endovascular versus surgical treatment in patients with carotid stenosis in the Carotid and Vertebral Artery Transluminal Angioplasty Study (CAVATAS): a randomised trial. Lancet $2001 ; 357: 1729-37$.
87. European Atrial Fibrillation Study Group. Secondary prevention in non-rheumatic atrial fibrillation after transient ischaemic attack or minor stroke. Lancet 1993; 34: 1255-62.

88. Labovitz AJ, for the STEPS Investigators. Transesophageal echocardiography and unexplained cerebral ischemia: a multicenter follow-up study. Am Heart J 1999; 137: 1082-7.

89. Nendaz MR, Sarasin FP, Junod AF, Bogousslavsky J. Preventing stroke recurrence in patients with patent foramen ovale: antithrombotic therapy, foramen closure, or therapeutic abstention? A decision analytic perspective. Am Heart J 1998; 135: 532-41.

90. Overwell JR, Bone I, Lees KR. Interatrial septal abnormalities and stroke. Neurology 2000; 55: 1172-9.

91. Mattioli AV, Aquilina M, Oldani A, Longhini C, Mattioli G. Atrial septal aneurysm as a cardioembolic source in adult patients with stroke and normal carotid arteries. A multicentre study. Eur Heart J 2001; 22: 261-8.

92. Homma S, Sacco RL, Di Tullio MR, Sciacca RR, Mohr JP, PFO in Cryptogenic Stroke Study (PICSS) Investigators. Effect of medical treatment in stroke patients with patent foramen ovale: patent foramen ovale in Cryptogenic Stroke Study. Circulation 2002; 105: 2625-31.

93. Mas JL, Arquizan C, Lamy C, Zuber M, Cabanes L, Derumeaux G, et al, Patent Foramen Ovale and Atrial Septal Aneurysm Study Group. Recurrent cerebrovascular events associated with patent foramen ovale, atrial septal aneurysm, or both. N Engl J Med 2001; 345: 1740-6.

94. Braun M, Gliech V, Boscheri A, Schoen S, Gahn G, Reichmann H, et al. Transcatheter closure of patent foramen ovale (PFO) in patients with paradoxical embolism. Periprocedural safety and mid-term follow-up results of three different device occluder systems. Eur Heart J 2004; 25 : 424-30.

95. Messé SR, Silverman IE, Kizer JR, Homma S, Zahn C, Gronseth G, et al. Practice parameter: recurrent stroke with patent foramen ovale and atrial septal aneurysm. Report of the quality standards subcommittee of the American Academy of Neurology. Neurology 2004; 62: 1042-50.

96. Benninger DH, Georgiadis D, Kremer C, Studer A, Nedeltchev K, Baumgartner RW. Mechanism of ischemic infarct in spontaneous carotid dissection. Stroke 2004; 35: 482-5.

97. Lyrer P, Engelter S. Antithrombotic drugs for carotid artery dissection. Stroke 2004; 35: 613-4.

98. Barinagarrementería F, González-Duarte A, Cantú-Brito C. Estados protrombóticos e isquemia cerebral. Rev Neurol 1998; 26: 85-91.

99. Larracoechea-Jausoro J. Enfermedades hematológicas y accidente cerebrovascular: anticuerpos antifosfolípidos e ictus. Neurología 1997; 12: $36-43$.

100. Dressler F, Craig W, Castello R, Labovitz A. Mobile aortic atheroma and systemic emboli: efficacy of anticoagulation and influence of plaque morphology on recurrent stroke. J Am Coll Cardiol 1998; 31: 134-8.

101. Echiverri H, Rubino F, Gupta S, Gujrati M. Fusiform aneurysm of the vertebrobasilar arterial system. Stroke 1989; 20: 1741-7.

3. ACTUACIÓN CLÍNICA EN EL ATAQUE ISQUÉMICO TRANSITORIO

1. Bruce OM, Kidwell CSM, Saver JL. Epidemiological impact in the United States of a tissue-based definition of transient ischaemic attack. Stroke 2003; 34: 919-24.

2. Weimar C, Kraywinkel K, Rödl J, Hippe A, Harms L, Kloth A, et al. Etiology, duration and prognosis of transient ischaemic attacks. An analysis from the German Stroke Data Bank. Arch Neurol 2002; 59: 1589-94.

3. Albers GW, Caplan LR, Easton JD, Fayad PB, Mohr JP, Saver JL, et al, for The TIA Working Group. Transient ischaemic attack -proposal for a new definition. N Engl J Med 2003; 347: 1316-713.

4. National Institute of Neurological Disorders and Stroke ad hoc Committee. Classification of cerebrovascular diseases III. Stroke 1990; 21: 637-76.

5. Feldmann E, Wilterdink J. The symptoms of transient cerebral ischaemic attacks. Sem Neurol 1991; 11: 135-45.

6. Brodtkorb E, Gimse R, Antonaci F, Ellertsen B, Sand T, Sulg I, et al. Hyperventilation syndrome: clinical ventilatory and personality characteristics as observed in the neurological practice. Acta Neurol Scand 1990; 81: 307-13.

7. Lee H, Lerner A. Transient inhibitory seizures mimicking crescendo TIAs. Neurology 1990; 40: 165-6.

8. Feinberg WM, Albers GW, Barnett HJM, Biller J, Caplan LR, Carter LP, et al. Guidelines for the management of transient ischaemic attacks: from the Ad Hoc Committee on Guidelines for the Management of Transient Ischaemic Attacks of the Stroke Council of the American Heart Association. AHA medical/scientific statement: special report. Circulation 1994; 89: 2950-65.

9. Kimura K, Minematsu K, Yasaka M, Wada K, Yamaguchi T. The duration of symptoms in transient ischaemic attack. Neurology 1999; 52: 976-80.

10. Brown RD, Evans BA, Wiebers DO, Petty GW, Meissner I, Dalle AJD. 
Transient ischaemic attack and minor ischaemic stroke: an algorithm for evaluation and treatment. Mayo Clin Proc 1994; 69: 1027-39.

11. Evans BA, Sicks JD, Whisnant JP. Factors affecting survival and occurrence of stroke in patients with transient ischaemic attacks. Mayo Clin Proc 1994; 69: 416-21.

12. Pessin MS, Duncan GW, Mohr JP, Poskanzer DC. Clinical and angiographic features of carotid transient ischaemic attacks. N Engl J Med 1977; 296: 358-62.

13. Bruno A, Corbett JJ, Biller J, Adams HP Jr, Qualls C. Transient monocular visual loss patterns and associated vascular abnormalities. Stroke 1990; 21: 34-9.

14. Baquis GD, Pessin MS, Scott RM. Limb-shaking -a carotid TIA. Stroke 1985; 16: 444-8.

15. Carolei A, Candelise L, Fiorelli M, Fieschi C. Long-term prognosis of transient ischaemic attacks and reversible ischaemic neurologic deficits: a hospital-based study. Cerebrovasc Dis 1992; 2: 266-72.

16. Dennis M, Bamford J, Sandercock P, Warlow C. Prognosis of transient ischaemic attacks in the Oxfordshire Community Stroke Project. Stroke 1990; 21: 848-53

17. Heyman A, Wilkinson WE, Hurwitz BJ, Haynes CS, Utley CM, Rosati RA. Risk of ischaemic heart disease in patients with TIA. Neurology 1984; 34: 626-30.

18. Dyken ML, Conneally M, Haerer AF, Gotshall RA, Calanchini PR, Poskanzer DC, et al. Cooperative study of hospital frequency and character of transient ischaemic attacks, I: background, organization and clinical survey. JAMA 1977; 237: 882-6.

19. Johnston SC, Grees DR, Browner WS, Sidney S. Short-term prognosis after emergency department diagnosis of TIA. JAMA 2000; 284: 2901-6.

20. Johnston SC, Smith WS. Practice variability in management of transient ischaemic attacks. Eur Neurol 1999; 42: 105-8

21. Dutch TIA Trial Study Group. Predictors of major vascular events in patients with a transient ischaemic attack or nondisabling stroke. Stroke 1993; 24: 527-31

22. Koudstaal PJ. Transient ischaemic attacks: diagnosis and prognosis. Cerebrovasc Dis 1994; 4 (Suppl 1): 40-6.

23. Davalos A, Matías-Guiu J, Torrent O, Vilaseca J, Codina A. Computed tomography in reversible ischaemic attacks: clinical and prognostic correlations in a prospective study. J Neurol 1988; 235: 155-8.

24. Landi G, Motto C, Cella E, Musicco M, Lipari S, Boccardi E, et al. Pathogenic and prognostic features of lacunar transient attack syndromes. J Neurol Neurosurg Psychiatry 1993; 56: 1265-70.

25. Alvarez-Sabín J. Accidente isquémico transitorio: ¿diagnóstico en régimen de hospitalización o ambulatorio? Rev Neurol 1997; 25: 1104-9.

26. Goldstein LB, Bian J, Samsa GP, Bonito AJ, Lux LJ, Matchar DB. New transient ischaemic attack and stroke: outpatient management by primary care physicians. Arch Intern Med 2000; 160: 2941-6.

27. European Carotid Surgery Trialists' Collaborative group. MRC European Carotid Surgery Trial: interim results for symptomatic patients with severe (70-99\%) or with mild (0-29\%) stenosis carotid. Lancet 1991; 337: 1235-43.

28. Carolei A, Marini C, Nencini P, Gandolfo C, Motto C, Zanette E, et al. Prevalence and outcome of symptomatic carotid lesions in young adults. Br Med J 1995; 310: 1363-6.

29. Bogousslavsky J, Hachinski VC, Boughner DR, Fox AJ, Viñuela F, Barnett HJ. Cardiac and arterial lesions in carotid ischaemic attacks. Arch Neurol 1986; 43: 223-8.

30. Amarenco P, Cohen A, Tzourio C, Bertrand B, Hommel M, Besson G, et al. Atherosclerotic disease of the aortic arch and the risk of ischaemic stroke. N Engl J Med 1994; 331: 1474-9.

31. Cerebral Embolism Task Force. Cardiogenic brain embolism. The second report of the Cerebral Embolism Task Force. Arch Neurol 1989; 46: 727-43.

32. Bogousslavsky J, Regli F. Delayed TIAs distal to bilateral occlusion of carotid arteries. Evidence for embolic and hemodynamic mechanisms. Stroke 1983; 14: 58-61.

33. Castillo J. Ataque isquémico transitorio. In Díez-Tejedor E, en nombre del comité de redacción ad hoc del Grupo de Estudio de Enfermedades Cerebrovasculares de la Sociedad Española de Neurología. Guía para el tratamiento y prevención del ictus. Neurología 1998; 13 (Suppl 3): 11-2.

34. Lees KR, Bath PMW, Naylor AR. ABC of arterial and venous disease. Secondary prevention of transient ischaemic attack and stroke. Br Med J 2000; 320: 991-4.

35. Anwer UE. Essential investigations of patients with suspected TIAs. Eur Neurol 1998; 39 (Suppl 1): 17-20.

36. Culebras A, Kase C, Masdeu JC, Fox AJ, Bryan RN, Grossman CB, et al. Practice guidelines for the use of imaging in transient ischaemic attacks and acute stroke: a report of the Stroke Council, American Heart Association. Stroke 1997; 28: 1480-97.

37. Dennis M, Bamford J, Sandercock P, Molyneux A, Warlow C. Com- puted tomography in patients with transient ischaemic attacks: when is a transient ischaemic attack not a transient ischaemic attack but a stroke? J Neurol 1991; 237: 257-61.

38. Koudstaal PJ, Van Gijn J, Frenken CW, Hijdra A, Lodder J, Vermeulen $\mathrm{M}$, et al. TIA, RIND, minor stroke: a continuum, or different subgroups? Dutch TIA Study Group. J Neurol Neurosurg Psychiatry 1992; 55: 95-7.

39. Bhadelia R, Anderson M, Polak JF, Manolio TA, Beauchamp N, Knepper L, et al. Prevalence and association of MRI-demonstrated brain infarcts in elderly subjects with a history of transient ischaemic attack: the Cardiovascular Health Study. Stroke 1999; 30: 383-8.

40. Kidwell CS, Alger J, Di Salle F, Starkman S, Villablanca P, Bentson J, et al. Diffusion MRI in patients with transient ischaemic attacks. Stroke 1999; 30: 1174-80.

41. Baracchini C, Manara R, Ermani M, Meneghetti G. The quest for early predictors of stroke evolution: can TCD be a guiding light? Stroke 2000; 31: 2942-7.

42. Goertler M, Kross R, Baeumer M, Jost S, Grote R, Weber S, et al. Diagnostic impact and prognostic relevance of early contrast-enhanced transcranial color-coded duplex sonography in acute stroke. Stroke 1999; 30: 897-9.

43. Sorensen AG, Bounanno FS, González RG, Schwamm LH, Lev MH, Huang-Hellinger FR, et al. Hyperacute stroke: evaluation with combined multisection diffusion-weighted and hemodynamically weighted echo-planar MR imaging. Radiology 1996; 199: 391-401.

44. Cheitlin MD, Alpers JS, Armstrong WF, Aurigemma GP, Beller GA, Bierman FZ, et al. ACC/AHA guidelines for the clinical application of echocardiography: executive summary. A report of the American College of Cardiology/American Heart Association Task Force on Practice Guidelines (Committee on Clinical Application of Echocardiography). J Am Coll Cardiol 1997; 28: 862-79.

45. Gubitz G, Phillips S, Dwyer V. What is the cost of admitting patients with transient ischaemic attacks to hospital. Cerebrovasc Dis 1999; 9: 210-4.

46. Markus H, Culliname M. Severely impaired cerebrovascular reactivity predicts stroke and TIA risk in patients with carotid artery stenosis and occlusion. Brain 2001; 124: 457-67.

47. Valton L, Larrue V, Le Traon AP, Geraud G. Cerebral microembolism in patients with stroke or transient ischaemic attack as a risk factor for early recurrence. J Neurol Neurosurg Psychiatry 1997; 63: 784-7.

48. Henneman PL, Lewis RJ. Is admission medically justified for all patients with acute stroke or transient ischaemic attacks? Ann Emerg Med 1995; 25: 458-63.

49. Pop GA, Koudstaal PJ, Meeder HJ, Algra A, Van Latum JC, Van Gijn J. Predictive value of clinical history and electrocardiogram in patients with transient ischaemic attack or minor ischaemic stroke for subsequent cardiac and cerebral ischaemic events. The Dutch TIA Trial Study Group. Arch Neurol 1994; 51: 333-41.

50. Sacco RL, Benjamin EJ, Broderick JP, Dyken M, Easton JD, Feinberg WM, et al. Risk factors panel: American Heart Association Prevention Conference IV: prevention and rehabilitation of stroke: risk factors. Stroke 1997; 28: 1507-17.

51. Albers GW, Hart RG, Lutset HL, Newell DW, Sacco RL. Supplement to the guidelines for the management of transient ischaemic attacks. A statement from the Ad Hoc Committee on Guidelines for the management of transient ischaemic attacks, Stroke Council, American Heart Association. Stroke 1999; 30: 2502-11.

52. Stassen JA, Fagard R, Thijs L, Celis H, Arabidze GG, Birkenhager $\mathrm{WH}$, et al. Randomised double-blind comparison of placebo and active treatment for older patients with isolated systolic hypertension: the Systolic Hypertension in Europe (Syst-Eur) Trial Investigators. Lancet 1997; 350: 757-64.

53. International Society of hypertension (ISH). Statement on blood pressure lowering and stroke prevention. J Hypertens 2003; 21: 651-63.

54. European Society of Hypertension-European Society of Cardiology. Guidelines for the management of arterial hypertension. J Hypertens 2003; 21: 1011-53.

55. Chobanian AV, Bakris GL, Black HR, Cushman WC, Green LA, Izzo $\mathrm{JL} \mathrm{Jr}$, et al. The Seventh Report of the Joint Committee on prevention, detection, evaluation and treatment of high blood pressure. The JNV Seventh Report. JAMA 2003; 289: 2560-72.

56. Bautista A, Pérez GE. Evaluación y manejo actual de la hipertensión arterial esencial. Acta Neurológica Colombiana 2003; 19: 69-74.

57. Robbins AS, Manson JE, Lee IM, Satterfield S, Hennekens CH. Cigarette smoking and stroke in a cohort of US male physicians. Ann Intern Med 1994; 120: 458-62.

58. Engstad T, Viitanen M, Arnesen E. Predictors of death among longterm stroke survivors. Stroke 2003; 34: 2876-80.

59. Hebert PR, Gaziano JM, Chan KS, Hennekens CH. Cholesterol lowering with statin drugs, risk of stroke, and total mortality: an overview of randomized trials. JAMA 1997; 278: 313-21. 
60. The Long-Term Intervention with Pravastatin in Ischaemic Disease (LIPID) Study Group. Prevention of cardiovascular events and death with pravastatin in patients with coronary heart disease and a broad range of initial cholesterol levels. N Engl J Med 1998; 339: 1349-57.

61. Furberg CD, Adams HP, Applegate WB, Byington RP, Espeland MA Hartwell T, et al, for the Asymptomatic Carotid Artery Progression Study (ACAPS) Research Group. Effect of lovastatin on early carotid atherosclerosis and cardiovascular events. Circulation 1994; 90: 1679-87.

62. Crouse JR III, Byington RP, Bond MG, Espeland MA, Craven TE, Sprinkle JW, et al. Pravastatin, lipids, and atherosclerosis in the carotid arteries (Plac-II). Am J Cardiol 1995; 75: 455-9.

63. American Diabetes Association. Clinical practice recommendations 1998. Diabetes Care 1998; 21 (Suppl 1): S1-89.

64. Balkau B, Shipley M, Jarrett RJ, Pyorala K, Pyorala M, Forhan A, et al. High blood glucose concentration is a risk factor for mortality in middle-aged nondiabetic men: 20-year follow-up in the Whitehall Study, the Paris Prospective Study, and the Helsinki Policemen Study. Diabetes Care 1998; 21: 360-7.

65. Writing Group for the Women's Health Initiative Investigators. Risks and benefits of estrogen plus progestin in healthy postmenopausal women. Principal results from the Women's Health Initiative randomized controlled trial. JAMA 2002; 288: 321-33.

66. Nelson HD, Humphrey LL, Nygren P, Teustch SM, Allan JD. Postmenopausal hormone replacement therapy. Scientific review. JAMA 2002; 288: 872-81.

67. Collaborative meta-analysis of randomized trials of antiplatelet therapy for prevention of death, myocardial infarction, and stroke in high risk patients. Antithrombotic Triallists' Collaboration. Br Med J 2002; 324: 71-86.

68. Farrell B, Godwin J, Richards S, Warlow C. The United Kingdom Transient Ischaemic Attack (UK-TIA) aspirin trial: final results. J Neurol Neurosurg Psychiatry 1991; 54: 1044-54.

69. The Dutch TIA Trial Study Group. A comparison of two doses of aspirin (30mg vs $283 \mathrm{mg}$ a day) in patients after a transient ischaemic attack or minor ischaemic stroke. N Engl J Med 1991; 325: 1261-6.

70. Gent M, Blakely JA, Easton JD, Ellis DJ, Hachinski VC, Harbison JW, et al. The Canadian American Ticlopidine Study (CATS) in thromboembolic stroke. Lancet 1989; 1: 1215-20.

71. Hass WK, Easton JD, Adams HP Jr, Pryse-Phillips W, Molony BA, Anderson S, et al. A randomized trial comparing ticlopidine hydrochloride with aspirin for the prevention of stroke in high-risk patients: Ticlopidine Aspirine Stroke Study Group. N Engl J Med 1989; 321: 501-7.

72. CAPRIE Steering Committee. A randomised, blinded, trial of clopidogrel versus aspirin in patients at risk of ischaemic events (CAPRIE). Lancet 1996; 348: 1329-39.

73. Diener HC, Cunha L, Forbes C, Sivenius J, Smets P, Lowenthal A. European Stroke Prevention Study 2: dipyridamole and acetylsalicylic acid in the secondary prevention of stroke. J Neurol Sci 1996; 143: 1-13.

74. Matías-Guiu J, Ferro J, Álvarez-Sabin J. Triflusal versus Aspirin in secondary stroke prevention: results of TACIP study [abstract]. Stroke 2001; 32: 329b.

75. The Stroke Prevention in Reversible Ischemia Trial (SPIRIT) Study Group. A randomized trial of anticoagulants versus aspirin after cerebral ischaemia of presumed arterial origin. Ann Neurol 1997; 42: 857-65.

76. Mohr JP, Thompson JLP, Lazar RM. Levin B, Sacco RL, Furie KL, et al for the Warfarin-Aspirin Recurrent Stroke Study Group. A comparison of warfarin and aspirin for the presentation of recurrent ischemic stroke. N Engl J Med 2001; 345: 1444-51.

77. EAFT (European Atrial Fibrillation Trial) Study Group. Secondary prevention of vascular events in patients with nonrheumatic atrial fibrillation and a recent transient ischaemic attack or minor ischaemic stroke. Lancet 1993; 342: 1255-62.

78. Hart RG, Halperin JL. Atrial fibrillation and stroke. Concepts and controversies. Arch Neurol 2000; 57: 326-32

79. The North American Symptomatic Carotid Endarterectomy Trial Collaborators. Beneficial effect of carotid endarterectomy in symptomatic patients with high grade carotid stenosis. N Engl J Med 1991; 325: 325-453.

80. Barnett HJM, Taylor DW, Eliasziw M, Fox AJ, Ferguson GG, Haynes B, et al, for the North American Symptomatic Carotid Endarterectomy Trial Collaborators. Benefit of carotid endarterectomy in patients with symptomatic moderate or severe stenosis. N Engl J Med 1998; 339: 1415-25.

81. European Carotid Surgery Trialists' Collaborative Group (CEST). Randomised trial of endarterectomy for recently symptomatic carotid stenosis: final results of the MCR European Carotid Surgery Trial (ECST). Lancet 1998; 351: 1379-87.

82. Rothwell PM, Warlow CP. Prediction of benefit from carotid endarterectomy in individual patients: a risk modeling study. Lancet 1999; 353: $2105-7$.
83. Barnett H, Meldrum HE. Carotid endarterectomy a neurotherapeutic advance. Arch Neurol 2000; 57: 40-5.

84. Kistler JP, Furie KL. Carotid endarterectomy revisited. N Engl J Med 2000; 342: 1693-700.

85. Goldstein LB, Samsa GP, Matchar DB, Oddone EZ. Multicenter review of preoperative risk factors for endarterectomy for asymptomatic carotid artery stenosis. Stroke 1998; 29: 750-3.

86. Chaturvedi S, Aggarwal Murugappan A. Results of carotid endarterectomy with prospective neurologist follow up. Neurology 2000; 55: 769-72.

87. Paciaroni M, Eliasziw M, Sharpe BL, Kappelle J, Chaturvedi S, Meldrum $\mathrm{H}$, et al. Long-term clinical and angiographic outcomes in symptomatic patients with $70 \%$ to $99 \%$ carotid artery stenosis. Stroke 2000 31: 2037-42.

88. Barnett H, Meldrum HE. Endarterectomy for carotid stenosis: new approaches in patient selection. Cerebrovasc Dis 2001; 11 (Suppl 1): 105-11.

89. Kappelle LJ, Eliasziw M, Fox AJ, Sharpe BL, Barnett HJM, for the North American Symptomatic Carotid Endarterectomy Trial (NASCET) Group. Importance of intracranial atherosclerotic disease in patients with symptomatic stenosis of the internal carotid artery. Stroke 1999; 30: 282-6.

90. Bamford J. Risk stratification and carotid surgery: new technology but old trials. Brain 2001; 124: 455-6.

91. Gil-Peralta A, Mayol A, Marcos JR, González A, Ruano J, Boza F, et al. Percutaneous transluminal angioplasty of the symptomatic atherosclerotic carotid arteries. Results, complications, and follow-up. Stroke 1996; $27: 2271-3$

92. Malek AM, Higashida RT, Phatouros CC, Lempert TE, Meyers PM, Smith WS, et al. Stent angioplasty for cervical carotid artery stenosis in highrisk symptomatic NASCET-ineligible patients. Stroke 2000; 31: 3029-33.

4. MANEJo DEL INFARTo CEREBRAL AGUdo

1. Egido JA, Alonso de Leciñana M, Martínez-Vila E, Díez-Tejedor E, en representación del comité ad hoc del Grupo de Estudio de Enfermedades Cerebrovasculares de la Sociedad Española de Neurología. Guía para el tratamiento del infarto cerebral agudo. Neurología 2002; 17 (Suppl 3): 43-60.

2. Adams HP, Adams JR, Brott T, Del Zoppo GJ, Furlan MD, Goldstein $\mathrm{MD}$, et al. Guidelines for the early management of patients with ischemic stroke. A scientific statement from the stroke council of the American Stroke Association. Stroke 2003; 34: 1056-83.

3. European Stroke Initiative Executive Committee and Writing Committee. The European stroke initiative recommendations for stroke management. Update 2003. Cerebrovasc Dis 2003; 16: 311-8.

4. Klijn CJM, Hankey GJ. Management of acute ischemic stroke: new guidelines from the American Stroke Association and European Stroke Initiative. Lancet Neurol 2003; 2: 698-791.

5. Dávalos A, Castillo J, Martínez-Vila E, for the Cerebrovascular Disease Study Group of the Spanish Society of Neurology. Delay in neurological attention and stroke outcome. Stroke 1995; 26: 2233-7.

6. Smith MA, Shahar E, McGovern PG, Kane RL, Doliszny KM, Arnett DK, et al. HMO membership and patient age and the use of specialty care for hospitalized patients with acute stroke: the Minnesota Stroke Survey. Med Care 1999; 37: 1186-98.

7. Lanska DJ, and the Task Force on Hospital Utilization for Stroke of the American Academy of Neurology. Review criteria for hospital utilization for patients with cerebrovascular disease. Neurology 1994; 44: 1531-2.

8. Indredavik B, Bakke F, Solberg R, Rokseth R, Haaheim LL, Holme I. Benefit of a stroke unit: a randomized controlled trial. Stroke 1991; 22: 1026-31.

9. Egido JA, González JL, Varela de Seijas E. Experiencia de una unidad de ictus en el hospital clínico de madrid. Rev Neurol 1995; 23: 381-4.

10. Díez-Tejedor E, Fuentes B. Acute care in stroke: do stroke units make the difference? Cerebrovasc Dis 2001; 11 (Suppl 1): 31-9.

11. European Federation of Neurological Societies Task Force. Neurological acute stroke care: the role of European neurology. Eur J Neurol 1997; 4: 435-41.

12. Roming OM, Guldvog B. Should stroke victims routinely receive supplemental oxygen? A quasi-randomized controlled trial. Stroke 1999; 30: 2033-7.

13. Steiner T, Mendoza G, De Georgia M, Schellinger P, Holle R, Hacke W. Prognosis of stroke patients requiring mechanical ventilation in a neurological critical care unit. Stroke 1997; 28: 7115.

14. Powers WJ. Acute hypertension after stroke: the scientific basis for treatment decisions. Neurology 1993; 43: 4617.

15. Vemmos KN, Tsivgoulis G, Spengos K, Zakopoulos N, Synetos A, Manios E, et al. U-shaped relationship between mortality and admission blood pressure in patients with acute stroke. J Intern Med 2004; 255: $257-65$ 
16. Castillo J, Leira R, García MM, Serena J, Blanco M, Dávalos A. Blood pressure decrease during the acute phase of ischemic stroke is associated with brain injury and poor stroke outcome. Stroke 2004; 35: 520-7.

17. Adams HP, Brott T, Furlan AJ, Gómez CR, Grotta J, Helgason CM, et al. Guidelines for thrombolytic therapy for acute stroke: A supplement to the guidelines for the management of patients with acute ischemic stroke. A statement for healthcare professionals from a special writing group of the stroke council, AHA. Stroke 1996; 27: 1711-8.

18. Practice advisory: thrombolytic therapy for acute ischemic stroke -summary statement. Report of the Quality Standards Subcommittee of the American Academy of Neurology. Neurology 1996; 47: 835-9.

19. Hajat C, Hajat S, Sharma P. Effects of poststroke pyrexia on stroke outcome: a meta-analysis of studies in patients. Stroke 2000; 31: 404-9.

20. Dippel DW, Van Breda EJ, Van Gemert HM, Van der Worp HB, Meijer RJ, Kappelle LJ, et al. Effect of paracetamol (acetaminophen) on body temperature in acute ischemic stroke: a double-blind, randomized phase II clinical trial. Stroke 2001; 32: 1607-12.

21. Weir CJ, Murray GD, Dyker AG, Lees KR. Is hyperglycaemia an independent predictor of poor outcome after acute stroke? Results of a longterm follow up study. Br Med J 1997; 314: 1303-6.

22. Gray CS, Hildreth AJ, Alberti GK, O'Connell JE; GIST Collaboration. Poststroke hyperglycemia: natural history and immediate management. Stroke 2004; 35: 122-6.

23. Bath PM, Bath FJ, Smithard DG. Interventions for dysphagia in acute stroke. Cochrane Database Syst Rev 2000; 2: CD000323.

24. Norton B, Homer-Ward M, Donelly MT, Long RG, Holmes GK. A randomised prospective comparison of percutaneous endoscopic gastrostomy and nasogastric tube feeding after acute dysphagic stroke. $\mathrm{Br}$ Med J 1996; 312: 13-6.

25. Gariballa SE, Parker SG, Taub N, Castleden CM. Influence of nutritional status on clinical outcome after acute stroke. Am J Clin Nutr 1998; 68: 375-81.

26. Langhorne P. Measures to improve recovery in the acute phase of stroke. Cerebrovasc Dis 1999; 9 (Suppl 5): 2-5.

27. Sunderland A, Tinson DJ, Bradley EL, Fletcher D, Langton HR, Wade DT. Enhanced physical therapy improves recovery of arm function after stroke. A randomised controlled trial. J Neurol Neurosurg Psychiatry $1992 ; 55: 530-5$.

28. Kwakkel G, Wagenaar RC, Koelman TW, Lankhorst GJ, Koetsier JC. Effects of intensity of rehabilitation after stroke. A research synthesis. Stroke 1997; 28: 1550-6.

29. Langhorne P, Wagenaar R, Partridge C. Physiotherapy after stroke: more is better? Physiother Res Int 1996; 1: 75-88.

30. De Pedro-Cuesta J, Widen-Holmqvist L, Bach-y-Rita P. Evaluation of stroke rehabilitation by randomized controlled studies: a review. Acta Neurol Scand 1992; 86: 433-9.

31. Ropper AH, Shafran B. Brain edema after stroke. Arch Neurol 1984; 41: 26-9.

32. Schneider GH, Von Helden GH, Franke R. Influence of body position on jugular venous oxygen saturation, intracranial pressure and cerebral perfusion pressure. Acta Neurochir 1993; (Suppl 59): 107.

33. Bauer RB, Téllez H. Dexamethasone as treatment in cerebrovascular disease. A controlled study of acute cerebral infarction. Stroke 1973; 4: 547-55.

34. Norris JW, Hachinski V. High dose steroid treatment in cerebral infarction. Br Med J 1986; 292: 21-3.

35. Schwab S, Spranger M, Schwarz S, Hacke W. Barbiturate coma in severe hemispheric stroke: useful or obsolete? Neurology 1997; 48: 1608-13.

36. Bereczki D, Liu M, Prado GF, Fekete I. Cochrane report: a systematic review of manitol therapy for acute ischemic stroke and cerebral parenchymal hemorrhage. Stroke 2000; 31: 2719-22.

37. Bayer AJ, Pathy MSJ, Newcombe R. Double blind randomized trial of intravenous glycerol in acute stroke. Lancet 1987; 1: 405-8.

38. Uhl E, Kreth FW, Elias B, Goldammer A, Hempelmann RG, Liefner $\mathrm{M}$, et al. Outcome and prognostic factors of hemicraniectomy for space occupying cerebral infarction. J Neurol Neurosurg Psychiatry 2004; 75: 270-4.

39. Rieke K, Krieger D, Adams HP, Aschoff A, Meyding-Lamade U, Hacke W. Therapeutic strategies in space occupying cerebellar infarction based on clinical, neurorradiological and neurophysiological data. Cerebrovasc Dis 1993; 3: 45-55

40. Reith J, Jorgensen HS, Nakayama H, Raaschou HO, Olsen TS. Seizures in acute stroke: predictors and prognostic significance. The Copenhagen Stroke Study. Stroke 1997, 28: 1585-9.

41. Bladin CF, Alexandrov AV, Bellavance A, Bornstein N, Chambers B, Cote R, et al. Seizures after stroke: a prospective multicenter study. Arch Neurol 2000; 57: 1617-22.

42. Egido JA, Alonso de Leciñana M. Riesgo cardiovascular en pacientes que han sufrido un evento cerebrovascular. Cuadernos Latinoamericanos de Hipertensión 2000; 5: 129-38.

43. Turpie ACG, Levin NM, Hirsh J, Carter CJ, Jay RM, Powers PJ, et al. A doubleblind randomized trial of Org 10172 low molecular weight heparinoid in prevention of deep vein thrombosis in thrombotic stroke. Lancet 1987; 1: 5236.

44. Turpie ACG, Gent M, Cote R, Levine MN, Ginsberg JS, Powers PJ, et al. A low molecular weight heparinoid compared with unfractioned heparin in the prevention of deep vein thrombosis in patients with acute ischemic stroke. A randomized, doubleblind study. Ann Intern Med 1992; 117: 3537

45. Bath PM, Iddenden R, Bath FJ. Low-molecular-weight heparins and heparinoids in acute ischemic stroke: a meta-analysis of randomized controlled trials. Stroke 2000; 3: 1770-8

46. International Stroke Trial Collaborative Group. The International Stroke Trial (IST): a randomized trial of aspirin, subcutaneous heparin, both or neither among 19435 patients with acute ischaemic stroke. Lancet 1997; 349: $1569-81$.

47. CAST (Chinese Acute Stroke Trial) Collaborative Group. CAST: randomized placebo controlled trial of early aspirin use in 20000 patients with acute ischaemic stroke. Lancet 1997; 349: 16419.

48. Antiplatelet Trialists' Collaboration. Collaborative overview of randomized trials of antiplatelet trials III: reduction in venous thrombosis and pulmonary embolism by antiplatelet prophylaxis among surgical and medical patients. Br Med J 1994; 308: 235-46.

49. Kamran SI, Downey D, Ruff L. Pneumatic sequential compression reduces the risk of deep venous thrombosis in stroke patients. Neurology 1998; 50: 1683-8.

50. Zheng MC, Sandercock P, Hong CP, Counsell C, Collins R, Li SL, et al. Indications for early aspirin use in acute ischemic stroke: a combined analysis of 40,000 randomized patients from the Chinese Acute Stroke Trial and the International Stroke Trial. Stroke 2000; 31: 1240-9.

51. The Publications Committee for the Trial of ORG 10172 in Acute Stroke Treatment (TOAST) Investigators. Low molecular weight heparinoid, ORG 10172 (danaparoid), and outcome after acute ischemic stroke: a randomized controlled trial. JAMA 1998; 279: 1265-72.

52. Hommel M, for the FISS Investigators group. Fraxiparine in Ischemic Stroke Study (FISS bis). Cerebrovasc Dis 1998; 8 (Suppl 4): 1-103.

53. Berge E, Abdelnoor M, Nakstad PH, Sandset PM, on behalf of the HAEST Study Group. Low-molecular weight heparin versus aspirin in patients with acute ischemic stroke and atrial fibrillation. A doubleblind randomised study. Lancet 2000; 355: 1205-10.

54. Diener HC, Ringelstein EB, Von Kummer R, Langohr HD, Bewermeyer $\mathrm{H}$, Landgraf $\mathrm{H}$, et al. Treatment of acute ischemic stroke with the low-molecular-weight heparin certoparin: results of the TOPAS trial. Therapy of Patients With Acute Stroke (TOPAS) Investigators. Stroke 2001; 32: 22-9.

55. Berge E, Sandercock P. Anticoagulants versus antiplatelet agents for acute ischemic stroke. Stroke 2003; 34: 1571-2.

56. The National Institute of Neurological Disorders and Stroke rtPA Stroke Study Group. Tissue plasminogen activator for acute ischemic stroke. N Engl J Med 1995; 333: 1581-7.

57. Hacke W, Kaste M, Fieschi C, Toni D, Lesaffre E, Von Kummer R, et al, for the ECASS Study Group. Intravenous thrombolysis with recombinant tissue plasminogen activator for acute hemispheric stroke. The European Cooperative Acute Stroke Study (ECASS). JAMA 1995; 274: 1017-25.

58. Hacke W, Kaste M, Fieschi C, Von Kummer R, Dávalos A, Meier D, et al. Randomised doubleblind placebocontrolled trial of thrombolytic therapy with intravenous alteplase in acute ischaemic stroke (ECASS II). Lancet 1998; 352: 1245-51.

59. Hacke W, Brott T, Caplan L, Meier D, Fieschi C, Von Kummer R, et al. Thrombolysis in acute ischemic stroke: controlled trials and clinical experience. Neurology 1999; 53 (Suppl 4): S3-14.

60. Marler JR, Tilley BC, Lu M, Brott TG, Lyden PC, Grotta JC, et al. Early stroke treatment associated with better outcome: the NINDS rtPA Stroke Study. Neurology 2000; 55: 1649-55.

61. Clark WM, Wissman S, Albers GW, Jhamandas JH, Madden KP, Hamilton S, for the ATLANTIS study investigators. Recombinant tissue-type plasminogen activator (alteplase) for ischemic stroke 3 to 5 hours after symptom onset. The ATLANTIS study: a randomized controlled trial. JAMA 1999; 282: 2019-26.

62. Hacke W, Donnan G, Fieschi C, Kaste M, Von Kummer R, Broderick JP, et al, ATLANTIS Trials Investigators, ECASS Trials Investigators, NINDS rt-PA Study Group Investigators. Association of outcome with early stroke treatment: pooled analysis of ATLANTIS, ECASS, and NINDS rt-PA stroke trials. Lancet 2004; 363: 768-74.

63. SITS-MOST protocol. URL: http://www.acutestroke.org.

64. Multicenter Acute Stroke Trial-Italy Group. Randomised controlled 
trial of streptokinase, aspirin, and combination of both in treatment of acute ischemic stroke. Lancet 1995; 346: 1509-14.

65. Ciccone A, Motto C, Aritzu E, Piana A, Candelisse L on behalf of the MAST-I Collaborative Group. Risk of aspirin use plus thrombolysis after acute ischemic stroke: a further MAST-I analyisis. Lancet 1998; 352: 880 .

66. Hommel M, Boissel JP, Cornu C, Boutitie F, Lees KR, Besson G, et al, for the MAST study group. Termination of trial of streptokinase in severe acute ischemic stroke. Lancet 1994; 345: 57.

67. Donnan GA, Davis SM, Chambers BR, Gates PC, Hankey GJ, McNeill JJ, et al. Trials of streptokinase in severe acute ischemic stroke. Lancet 1995; 345: 578-9.

68. Donnan GA, Hommel M, Davis SM, McNeill JJ, for the steering committees of the ASK and MASTE trials. Streptokinase in acute ischemic stroke. Lancet 1995; 346: 56.

69. Atkinson RP. Ancrod in the treatment of acute ischaemic stroke. Drugs 1997; 54 (Suppl 3): 100-8

70. Sherman OG, Atkinson RP, Chippeudale T, Levin KA, Intrell N, Hsu $\mathrm{CY}$, et al, for STAT. Intravenous Ancrod for treatment of acute ischaemic stroke. The STAT Study: a randomized controlled trial. JAMA 2000; 283: 2395-403.

71. Orgogozo JM, Verstraete M, Kay R, Hennerici M, Lenzi GL. Outcomes of Ancrod in acute ischemic stroke. Independent Data and Safety Monitoring Board for ESTAT. Steering Committee for ESTAT. European Stroke Treatment with Ancrod Trial. JAMA 2000; 284: 1926-7.

72. The Abciximab in Ischemic Stroke Investigators. Abciximab in acute ischemic stroke. A randomized, double-blind, placebo-controlled, dose-escalation study. Stroke 2000; 31: 601-9.

73. Del Zoppo GJ, Higashida RT, Furlan AJ, Pessin MS, Rowley HA, Gent $\mathrm{M}$ and the PROACT investigators. (PROACT). A phase II randomized trial of recombinant prourokinase by direct arterial delivery in acute middle cerebral artery stroke. Stroke 1998; 29: 4-11.

74. Furlan A, Higashida R, Weschler L, Gent M, Rowley H, Kase C, et al. Intra-arterial prourokinase for acute ischemic stroke. The PROACT II study: a randomized controlled trial. JAMA 1999; 282: 2003-11.

75. IMS Study Investigators. Combined intravenous and intra-arterial recanalization for acute ischemic stroke: the Interventional Management of Stroke Study. Stroke 2004; 35: 904-11.

76. Clark WM, Warach SJ, Pettigrew LC, Gammans RE, Sabounjian LA, for the Citicoline Stroke Study Group. A randomized dose-response trial of citicoline in acute ischemic stroke patients. Neurology 1997; 49: 671-8.

77. Clark WM, Williams BJ, Selzer KA, Zweifler RM, Sabounjian LA Gammans RE. A randomized efficacy trial of citicoline in patients with acute ischemic stroke. Stroke 1999; 30: 2592-7.

78. Gammans RE, Sherman J, ECCO 2000 Investigators. ECCO 2000 study of citicoline for treatment of acute ischemic stroke: final results. $25^{\text {th }}$ International Stroke Conference. New Orleans. February 2000

79. Warach S, Pettigrew LC, Dashe JF, Pullicino P, Lefkowitz DM, Sabounjian LA, et al. Effect of citicoline on ischemic lesions as measured by diffusion-weighted magnetic resonance imaging. Citicoline 010 Investigators. Ann Neurol 2000; 48: 713-22.

80. Dávalos A, Castillo J, Álvarez-Sabín J, Secades JJ, Mercadal J, López $\mathrm{S}$, et al. Oral citicoline in acute ischemic stroke: an individual patient data pooling analysis of clinical trials. Stroke 2002; 33: 2850-7.

81. Castillo J, Álvarez-Sabín J, Dávalos A, Díez-Tejedor E, Lizasoain I, Martínez-Vila E, et al. Revisión de consenso. Neuroprotección farmacológica en la isquemia cerebral: ¿es todavía una opción terapéutica? Neurología 2003; 18: 368-84.

82. Pérez GE, Celis JI, Muñoz-Collazos M, Pedraza OL, Villa LA. Ataque cerebrovascular agudo. In Zurek R, ed. Consensos en Neurología. Guías de práctica Clínica. Asociación Colombiana de Neurología. Bogotá: Exlibris; 2001. p. 57-105.

\section{TRATAMIENTO DE LA TROMBOSIS VENOSA CEREBRAL}

1. Bousser MG. Cerebral venous thrombosis: nothing, heparin, or local thrombolysis? Stroke 1999; 30: 481-3.

2. Einhäupl KM, Villringer A, Meister W. Heparin treatment in sinus venous treatment thrombosis. Lancet 1991; 338: 597-600.

3. De Bruijn FTM, Stam J, for the Cerebral Venous Sinus Thrombosis Study Group. Randomized, placebo-controlled trial of anticoagulant treatment with low-molecular-weight heparin for cerebral sinus thrombosis. Stroke 1999; 30: 484-8.

4. Frey JL, Muro GJ, McDougall CG. Cerebral venous thrombosis: combined intrathrombus rtPA and intravenous heparin. Stroke 1999; 30: 489-94

5. Ferro JM, Correia M, Pontes C, Baptista MV, Pita F; Cerebral Venous Thrombosis Portuguese Collaborative Study Group (Venoport). Cerebral vein and dural sinus thrombosis in Portugal: 1980-1998. Cerebrovasc Dis 2001; 11: 177-82.
6. ACTUACIÓN CLÍNICA EN LA HEMORRAGIA CEREBRAL

1. Broderick J, Brott T, Tomsick T, Tew J, Duldner J, Huster G. Management of intracerebral hemorrhage in a large metropolitan population. Neurosurgery $1994 ; 34: 882-7$.

2. Broderick JP, Adams HP Jr, Barsan W, Feinberg W, Feldmann E, Grotta $\mathrm{J}$, et al. Guidelines for the management of spontaneous intracerebral hemorrhage. A statement for healthcare professionals from a special writing group of the Stroke Council, American Heart Association. Stroke 1999; 30: 905-15.

3. Kase C, Mohr J, Caplan L. Intracerebral hemorrhage. In Barnett H, Mohr J, Stein B, Yatsu F, eds. Stroke: pathophysiology, diagnosis, and management. New York: Churchill-Livingstone; 1992. p. 561-616.

4. Dul K, Drayer B. CT and RM imaging of intracerebral hemorrhage. In Kase CS, Caplan LR, eds. Intracerebral hemorrhage. Vol 5. Boston, Mass: Butterworth-Heinemann; 1994. p. 73-93.

5. Lainez JM, Pareja A. Martí-Fábregas J, Leira R. Guía de actuación clínica en la hemorragia cerebral. Neurología 2002; 17 (Suppl 3): 76-86.

6. Hapin SF, Britton JA, Byme JV Clifton A, Hart G, Moore A. Prospective evaluation of cerebral angiography and computed tomography in cerebral haematoma. J Neurol Neurosurg Psychiatry. 1994; 57: 1180-6.

7. Zhu XL, Chan MSY, Poon WX. Spontaneous intracraneal haemorrhage: which patients need diagnostic cerebral angiography? A prospective study of 206 cases and review of the literature. Stroke 1997; 28: 1406-9.

8. Díez-Tejedor E, Fuentes B. Acute care in stroke: do stroke units make the difference? Cerebrovasc Dis 2001; 11: 31-9.

9. Kazui S, Minematsu K, Yamamoto H, Sawada T, Yamaguchi T. Predisposing factors to enlargement of spontaneous intracerebral hematoma. Stroke 1997; 28: 2370-5.

10. Powers WJ, Zazulia AR, Videen TO, Adams RE, Yundt KD, Aiyagari $\mathrm{V}$, et al. Autoregulation of cerebral blood flow surrounding acute (6 to 22 hours) intracerebral hemorrhage. Neurology 2001; 57: 18-24.

11. Morgenstern LB, Yonas H. Lowering blood pressure in acute intracerebral hemorrhage safe, but will it help? Neurology 2001; 57: 5-6.

12. Kase CS, Crowell RM. Prognosis and treatment of patients with intracerebral hemorrhage. In Kase C, Kaplan LR, eds. Intracerebral hemorrhage. 1 ed. Newton: Butterworth-Heinemann; 1994. p. 467-89.

13. Ginsberg MD, Busto R. Combating hipertermia in acute stroke: a significant clinical concern. Stroke 1998; 29: 529-34.

14. Cervoni L, Artico M, Salvati M, Bristot R, Franco C, Delfín R. Epileptic seizures in intracerebral hemorrhage: a clinical and prognostic study of 55 cases. Neurosurg Rev 1994; 17: 185-8.

15. Qureshi AI, Geocadin RG, Suarez JI, Ulatowski JA. Long-term outcome after medical reversal of transtentorial herniation in patients with supratentorial mass lesions. Crit Care Med 2000; 28: 1556-64.

16. Poungvarin N, Bhoopat W, Viriyavejakul A, Rodprasert P, Buranasiri P, Sukondhabhant $\mathrm{S}$, et al. Effects of dexamethasone in primary supratentorial intracerebral hemorrhage. N Engl J Med 1987; 316: 1229-33.

17. Duff TA, Ayeni S, Levin AB, Javid M. Nonsurgical management of spontaneous intracerebral hematoma. Neurosurgery 1981; 9: 387-93.

18. James HE, Lanfitt TW, Kumar V, Ghostine SY. Treatment of intracranial hypertension. Analysis of 105 consecutive continuous recordings of intracranial pressure. Acta Neurochir 1977; 36: 189-200.

19. Juvela S, Heisskanen O, Poramen A, Valtonen S, Kuurne T, Kaste M, et al. The treatment of spontaneous intracranial hemorrhage. A prospective randomized trial of surgical and conservative treatment. J Neurosurg 1989; 70: 755-8.

20. Auer LM, Deinsberger W, Niederkorn K, Gell G, Kleinert R, Schneider $\mathrm{G}$, et al. Endoscopic surgery versus medical treatment for spontaneous intracerebral hematoma: a randomized study. J Neurosurg 1989; 70: $530-5$.

21. Hankey GJ, Hon C. Surgery for primary intracerebral hemorrhage: is it safe and effective? A systematic review of case series and randomized trials. Stroke 1997; 28: 2126-32.

22. Prasad K, Shrivastava A. Surgery for primary supratentorial intracerebral hemorrhage (Cochrane review). In: Cochrane library, issue 2. Oxford: Update software; 2000.

23. Prasad K, Browman G, Srivastava A, Menon G. Surgery in primary supratentorial intracerebral hematoma: a meta-analysis of randomized trials. Acta Neurol Scand 1997; 95: 103-10.

24. Kase C. Cerebellar hemorrhage. In Kase C, Kaplan LR, eds. Intracerebral haemorrhage. 1 ed. Newton: Butterworth-Heinemann; 1994. p. 425-45.

25. Kobayashi S, Sato A, Kageyama Y, Nakamura H, Watanabe Y, Yamaura A. Treatment of hypertensive cerebellar haemorrhage, surgical or conservative management? Neurosurgery 1994; 34: 246-50.

26. Batjer HH, Reisch JS, Allen JS, Plaizier JL, Su CJ. Failure of surgery to improve outcome in hypertensive putaminal hemorrhage. A prospective randomized trial. Arch Neurol 1990; 7: 1103-6.

27. Kanno T, Sano H, Shinomiya Y, Katada K, Nagata J, Hoshino M, et al. 
Role of surgery in hypertensive intracranial hematoma. A comparative study of 305 nonsurgical and 154 surgical. J Neurosurg 1984; 61: 1091-9.

28. Coraddu M, Nurchi GL, Floris F, Meledu V. Considerations about the surgical indication of the spontaneous cerebral hematomas. J Neurol Sci 1990; 34: 35-9.

29. Neau JP, Ingrand P, Couderp C, Rosier MP, Bailbe M, Dumas P, et al. Recurrent intracerebral hemorrhage. Neurology 1997; 49: 106-13.

30. Hypertension Detection and Follow-up Program Cooperative Group. Five-years findings of the Hypertension Detection and Follow-up Program III. Reduction in stroke incidence among persons with high blood pressure. JAMA 1982; 247: 633-8.

31. Bae H, Jeong D, Lee K, Yung Y, Byun B. Recurrence of bleeding in patients with hypertensive intracerebral haemorrhage. Cerebrovasc Dis 1999; 9: 102-8

32. Kondziolka D, Lunsford LD, Flickinger JC, Kestle JR. Reduction of haemorrhage risk after stereotactic radiosurgery for cavernous malformations J Neurosurg 1995; 83: 825-31.

33. Karlsson B, Kihlstrom L, Lindquist C, Ericson K, Steiner L. Radiosurgery for cavernous malformations. J Neurosurg 1998; 88: 293-7.

34. Stein BM. Surgical decisions in vascular malformations of the brain. In Barnett JM, Mohr JP, Stein BM, Yatsu FM. Stroke pathophysiology, diagnosis and management. 2 ed. New York: Churchill-Livingstone; 1992. p. $1093-143$.

35. Karlsson B, Lindquist C, Steiner L. Prediction of obliteration after gamma knife surgery for cerebral arteriovenous malformations. Neurosurgery $1997 ; 40: 425-30$.

36. Adams HP, Brott T, Furlan AJ, Gómez CR, Grotta J, Helgason CM, et al. Guidelines for thrombolytic therapy for acute stroke: a supplement to the guidelines for the management of patients with acute ischemic stroke. A statement for healthcare professionals from a special writing group of the Stroke Council, AHA. Stroke 1996; 27: 1711-8.

\section{MANEJo CLÍNICO DE LA HEMORRAGIA SUBARACNOIDEA}

1. Vermeulen M, Van Gijn J. The diagnosis of subarachnoid hemorrhage. J Neurol Neurosurg Psychiatry 1990; 53: 365-72.

2. Atlas SW. MR imaging is highly sensitive for acute subarachnoid hemorrhage. Radiology 1993; 186: 319-22.

3. Mitchell P, Wilkinson ID, Hoggard N, Paley MN, Jellinek DA, Powell $\mathrm{T}$, et al. Detection of subarachnoid haemorrhage with magnetic resonance imaging. J Neurol Neurosurg Psychiatry 2001; 70: 205-11.

4. Weismann M, Mayer TE, Medele R, Bruckmann H. Diagnosis of acute subarachnoid hemorrhage at 1.5 tesla using proton-density weighted FSE and MRI sequences. Radiology 1999; 39: 860-5.

5. Jager HR, Mansmann U, Hausmann O, Partzsch U, Moseley IF, Taylor WJ. MRA versus digital subtraction angiography in acute subarachnoid haemorrhage: a blinded multireader study of prospectively recruited patients. Neuroradiology 2000; 42: 313-26.

6. Kaim A, Proske M, Kirsch E, Von Weymarn A, Radü EW, Steinbrich $\mathrm{W}$. Value of repeat-angiography in cases of unexplained subarachnoid hemorrhage. Acta Neurol Scand 1996; 93: 366-73.

7. Sloan MA, Haley EC Jr, Kassell NF, Henry ML, Stewart SR, Beskin $\mathrm{RR}$, et al. Sensitivity and specificity of transcranial Doppler ultrasonography in the diagnosis of vasospasm following subarachnoid hemorrhage. Neurology 1989; 39: 1514-8.

8. ISUIA Investigators. Unruptured intracranial aneurysms: risk of rupture and risk of surgical intervention. N Engl J Med 1998; 339: 1725-33.

9. Vivancos J, Rubio F, Soler L, Vila N. Guía de actuación clínica en la hemorragia subaracnoidea. Neurología 2002; 17 (Suppl 3): 87-105.

10. Higashida RT, Halbach VV, Dowd CF, Hieshima GB. Intracranial aneurysm: evolution and future role of endovascular techniques. Neurosurg Clin N Am 1994; 5: 413-25.

11. Johnston SC, Dudley RA, Gress DR, Ono L. Surgical and endovascular treatment of unruptured cerebral aneurysms at university hospitals. Neurology 1999; 52: 1799-1805.

12. Johnston SC, Wilson CB, Halbach VV, Higashida RT, Dowd CF, Mc Dermott MW, et al. Endovascular and surgical treatment of unruptured cerebral aneurysms: comparison risks. Ann Neurol 2000; 48: 11-9.

13. Locksley HB. Natural history of subarachnoid hemorrhage, intracranial aneurysm and arteriovenous malformations. J Neurosurg 1966; 25: 321-68.

14. Bederson JB, Awad IA, Wiebers DO, Piepgras D, Clarke-Haley E, Brott $\mathrm{T}$, et al. Recommendations for the management of patients with unruptured intracranial aneurysms. A statement for healthcare professionals from the Stroke Council of the American Heart Association. Circulation 2000; 102: 2300-8.

15. The Magnetic Resonance Angiography in Relatives of Patients with Subarachnoid Hemorrhage Study Group. Risk and benefits of screening for intracranial aneurysms in first-degree relatives of patients with sporadic subarachnoid hemorrhage. N Engl J Med 1999; 341: 1344-50.
16. Adams HP Jr, Love BB. Medical management of aneurysmal subarachnoid hemorrhage. In Barnett H, Mohr J, Stein B, Yatsu F, eds. Stroke. Vol. III. 2 ed. New York: Churchill-Livingstone; 1992. p. 1029-54.

17. Mayberg MR, Batjer H, Dacey R, Diringer M, Haley EC, Heros RC, et al. Guidelines for the management of aneurysmal subarachnoid hemorrhage. Stroke 1994; 25: 2315-28.

18. Park BE. Spontaneous subarachnoid hemorrhage complicated by communicating hydrocephalus: epsilon amino caproic acid as a possible predisposing factor. Surg Neurol 1979; 11: 73-80.

19. Kassell NF, Torner JC, Adams HP Jr. Antifibrinolytic therapy in the acute period following aneurysmal subarachnoid hemorrhage: preliminary observations from the Cooperative Aneurysm Study. J Neurosurg 1984; 61: 225-30.

20. Vermeulen M, Lindsay KW, Murray GD, Cheah F, Hijdra A, Muizelaar JP, et al. Antifibrinolytic treatment in subarachnoid hemorrhage. $\mathrm{N}$ Engl J Med 1984; 311: 432-7.

21. Adams HP Jr, Nibbelink DW, Torner JC, Sahs AL. Antifibrinolytic therapy in patients with aneurysmal subarachnoid hemorrhage: a report of the Cooperative Aneurysm Study. Arch Neurol 1981; 38: 25-9.

22. Pinna G, Pasqualin A, Vivenza G, Da-Pian R. Rebleeding, ischaemia and hydrocephalus following antifibrinolytic treatment for ruptured cerebral aneurysm: a retrospective clinical study. Acta Neurochir Wien 1988; 93: 77-87.

23. Roos Y, for the STAR study group. Antifibrinolytic treatment in subarachnoid hemorrhage: a randomized placebo-controlled trial. Neurology 2000; $54: 77-82$

24. Kasselll NF, Sasaki T, Colohan ART, Nazar G. Cerebral vasospasm following aneurysmal subarachnoid hemorrhage. Stroke 1985; 16: 562-72.

25. Hans P. Pharmacological therapeutic prospects of cerebral vasospasm. Ann Fr Anesth Reanim 1996; 15: 374-81.

26. Pasqualin A, Rosta L, Da Pian R, Cavazzani P, Scienza R. Role of computed tomography in the management of vasospasm after subarachnoid hemorrhage. Neurosurgery $1984 ; 15: 344-53$.

27. Awad IA, Carter LP, Spetzler RF, Medina M, Williams FC Jr. Clinical vasospasm after subarachnoid hemorrhage: response to hypervolemic hemodilution and arterial hypertension. Stroke 1987; 18: 365-72.

28. Kassell NF, Peerless SJ, Durward QJ, Beck DW, Drake CG, Adams HP Jr. Treatment of ischemic deficits from vasospasm with intravascular volume expansion and induced arterial hypertension. Neurosurgery 1982; 11: 337- 43

29. Lennihan L, Mayer SA, Fink ME, Beckford A, Paik MC, Zhang H, et al. Effect of hypervolemic therapy on cerebral blood flow after subarachnoid hemorrhage. A randomized controlled trial. Stroke 2000, 31: 383-91.

30. Haley EC, Kassell NF, Torner JC. A randomized controlled trial of high-dose intravenous nicardipine in aneurysmal subarachnoid hemorrhage: a report of the Cooperative Aneurysm Study. J Neurosurg 1993; 78: $537-47$

31. Allen GS, Ahn HS, Preziosi TJ, Battye R, Boone SC, Chou SN, et al Cerebral arterial spasm -a controlled trial of nimodipine in patients with subarachnoid hemorrhage. N Engl J Med 1983; 308: 619-24.

32. Mee E, Dorrance D, Lowe D, Neil-Dwyer G. Controlled study of nimodipine in aneurysm patients treated early after subarachnoid hemorrhage. Neurosurgery 1988; 22: 484-91.

33. Petruk KC, West M, Mohr G, Weir BK, Benoit BG, Gentili F, et al Nimodipine treatment in poor grade aneurysm patients. Results of a multicenter double-blind placebo-controlled trial. J Neurosurg 1988; 68: 505-17.

34. Pickard JD, Murray JD, Illingworth R, Shaw MD, Teasdale GM, Foy PM, et al. Effect of oral nimodipine on cerebral infarction and outcome after subarachnoid hemorrhage: British aneurysm nimodipine trial. $\mathrm{Br}$ Med J 1989; 298: 636-42.

35. Öhman J, Servo A, Heiskanen O. Long-term effects of nimodipine on cerebral infarcts and outcome after aneurysmal subarachnoid hemorrhage and surgery. J Neurosurg 1991; 74: 8-13.

36. Barker FG II, Ogilvy CS. Efficacy of prophylactic nimodipine for delayed ischemic deficit after subarachnoid hemorrhage: a metaanalysis. J Neurosurg 1996; 84: 405-14.

37. Roos YB, Levi M, Carrol TA, Beenen LF, Vermeulen M. Nimodipine increases fibrinolitic activity in patients with subarachnoid cerebral hemorrhage. Stroke 2001; 32: 1860-2.

38. Newell DW, Eskridge JM, Mayberg MR, Grady MS, Winn HR. Angioplasty for the treatment of symptomatic vasospasm following subarachnoid hemorrhage. J Neurosurg 1989; 71: 654-60.

39. Eskridge JM, Newell DW, Pendleton GA. Transluminal angioplasty for treatment of vasospasm. Neurosurg Clin N Am 1990; 1: 387-99.

40. Hasen D, Vermeulen M, Wijdicks EF, Hijdra A, Van Gijn J. Management problems in acute hydrocephalus after subarachnoid hemorrhage. Stroke 1989; 20: 747-53.

41. Van Gijn J, Hijdra A, Wijdicks EF, Vermeulen M, Van Crevel H. Acute 
hydrocephalus after aneurysmal subarachnoid hemorrhage. J Neurosurg 1985; 63: 355-62.

42. Rose FC, Sarner M. Epilepsy after ruptured intracranial aneurysm. Br Med J 1965; 1: 18-21.

43. Kvam DA, Loftus CM, Copeland B, Quest DO. Seizures during the immediate postoperative period. Neurosurgery 1983; 12: 14-7.

44. Johnston SC, Higashida RT, Barrow DL, Caplan LR, Dion JE, Hademenos G, et al. Recommendations for the endovascular treatment of intracranial aneurysms. Stroke 2002; 33: 2536-44.

45. Guglielmi G, Viñuela F, Sepetka I, Marcellari V. Electrothrombosis of sacular aneurysms via endovascular approach, part I: electrochemical basis, technique and experimental results. J Neurosurg 1991; 75: 1-7.

46. Guglielmi G, Viñuela F, Dion J, Duckwiler G. Electrothrombosis of sacular aneurysms via endovascular approach, part II: preliminary clinical experience. J Neurosurg 1991; 75: 8-14.

47. Bryan RN, Rigamonte D, Mathis JM. The treatment of acutely ruptured aneurysms: endovascular therapy versus surgery. AJNR Am J Neuroradiol 1997; 18: 1826-30

48. International Subarachnoid Aneurysm trial (ISAT) Collaborative Group. International Subarachnoid Aneurysm trial (ISAT) of neurosurgical clipping versus endovascular coiling in 2143 patients with ruptured intracranial aneurysms: a randomised trial. Lancet 2002; 360: 1267-74.

49. Bavinski G, Killer M, Ferraz-Leite H, Gruber A, Gross CE. Richling B. Endovascular therapy of idiopatic cavernous aneurysms over 11 years. AJNR Am J Neuroradiol 1998; 19: 559-65.

50. Casasco AK, Aymard A, Gobin YP, Houdart E, Rogopoulos A, George B, et al. Selective endovascular treatment of 71 intracranial aneurysms with platinum coils. J Neurosurg 1993; 79: 3-10.

51. Martin D, Rodesh G, Álvarez H, Lasjaunias P. Preliminary results of embolisation of non-surgical intracranial aneurysms with GD coils: the first year of their use. Neuroradiology 1996; 38: S142-50.

52. Debrun G, Fox A, Drake CG, Peerless S, Girvin J, Ferguson G. Giant unclippable aneurysms: treatment with detachable balloons. AJNR Am J Neuroradiol 1981; 2: 167-73.

53. Kuether TA, Nesbit GM, Barnwell SL. Clinical and angiographic outcomes, with treatment data, for patients with cerebral aneurysms treated with Guglielmi detachable coils. a single center experience. Neurosurgery $1998 ; 43$ : 1016-25.

54. Dovey Z, Misra M, Thornton J, Charbel FT, Debrun GM, Ausman JI. Guglielmi detachable coiling for intracranial aneurysms. The story so far. Arch Neurol 2001; 58: 559-64.

\section{RECOMENDACIONES PARA EL TRATAMIENTO Y PREVENCIÓN DEL ICTUS, 2004}

Resumen. Introducción. Según la Organización Mundial de la Salud, la enfermedad cerebrovascular constituye la tercera causa de muerte y la primera de invalidez en la población adulta en el mundo. Sin embargo, la atención urgente del paciente con ictus, en un centro hospitalario bien dotado de medios materiales y personales (neurólogos expertos) y la aplicación de un programa protocolizado de cuidados, métodos diagnósticos y tratamiento específico (unidades o equipos de ictus) mejora significativamente la evolución de los pacientes afectados. Desarrollo. Para difundir las evidencias científicas disponibles acerca del tratamiento y la prevención del ictus, un comité ad hoc de la Sociedad Iberoamericana de Enfermedad Cerebrovascular ha redactado esta guía, que recoge de manera esquemática todas las recomendaciones vigentes, con los niveles de evidencia que las respaldan y el grado de recomendación para cada caso. Conclusiones. Este documento pretende servir de apoyo y guía para el tratamiento y la prevención en los pacientes con ictus en todos los países de Iberoamérica, y contribuir a lograr los medios necesarios para mejorar la atención al paciente con ictus en las distintas administraciones sanitarias. [REV NEUROL 2004; 39: 465-86]

Palabras clave. Enfermedad cerebrovascular. Guía. Ictus. Protocolo. Recomendaciones. Sociedad Iberoamericana de Enfermedad Cerebrovascular.
55. Cognard C, Weill A, Rey A, Moret J. Intracranial berry aneurysms: angiographic and clinical results after endovascular treatment. Radiology 1998; 6: 499-510.

56. Saveland H, Hillman J, Brandt L, Edner G, Jakobsson KE, Algers C. Overall outcome in aneurysmal subarachnoid haemorrhage in a hospital population: a prospective study from neurosurgical units in Sweden during a year period. J Neurosurg 1992; 76: 729-34.

57. Seiler RW, Reulen HJ, Huber P, Grolimund P, Ebeling U, Steiger H. Outcome an aneurysmal subarachnoid in a hospital population: a prospective study including early operation, intravenous nimodipine and trancranial Doppler ultrasound. Neurosurgery 1988; 23: 598604.

58. Le Roux PD, Elliot JP, Downey L, Newell DW, Grady MS, Mayber J, et al. Improved outcome after rupture of anterior circulation aneurysms: a retrospective 10 year review of 224 good grade patients. J Neurosurg 1995; 83: 394-402

59. Kassell NF, Boarini DJ, Adams HP Jr, Sahs AL, Graf CJ, Torner JC, et al. Overall management of ruptured aneurysm: comparison of early and late operation. Neurosurgery 1981; 9: 120-8.

60. Whitfield PC, Moss H, O'Hare D, Smielewski P, Pickard JD, Kirkpathrick PJ. An audit of aneurysmal subarachnoid hemorrhage: earlier resuscitation and surgery reduces patient stay and deaths from rebleeding. J Neurol Neurosurg Psychiatry 1996; 60: 301-6.

61. Vanninen R, Koivisto T, Saari T, Herneniemi J, Vapalahti M. Ruptured intracranial aneurysms: acute endovascular treatment with electrolytically detachable coils. A prospective randomized study. Radiology 1999; 211: 325-36.

62. Raaymakers TW, Rinkel GJ, Limburg M, Algra A. Mortality and morbidity of surgery for unruptured intracranial aneurysms: a meta-analysis. Stroke 1998; 29: 1531-8.

63. Johnston SC, Shao S, Dudley RA, Berman MF, Gress DR. Treatment of unruptured cerebral aneurysms in California. Stroke 2001; 32: 597-605.

64. Lin T, Fox AJ, Drake CG. Regrowth of aneurysm sacs from residual neck following aneurysm clipping. J Neurosurg 1989; 70: 556-60.

65. Debrun GM, Aletich VA, Kehrli P, Misra M, Ausman JI, Charbel F. Selection of cerebral aneurysms for treatment using Guglielmi detachable coils: the preliminary University of Illinois at Chicago experience. Neurosurgery 1998; 43: 1281-95.

66. Raymond J, Roy D. Safety and efficacy of endovascular treatment of acutely ruptured aneurysms. Neurosurgery 1997; 41: 1235-46.

\section{RECOMENDAÇÕES PARA O TRATAMENTO E A PREVENÇÃO DO AVC, 2004}

Resumo. Introdução. Segundo a Organização Mundial da Saúde, a doença cérebro-vascular constitui a terceira causa de morte e a primeira de invalidez na população adulta mundialmente. No entanto, a atenção urgente para o doente com AVC, um centro hospitalar bem fornecido de meios materiais e humanos (neurologistas peritos) e a aplicação de um programa protocolizado de cuidados, métodos de diagnóstico e tratamento específico (unidades ou equipas de $A V C$ ), melhoraria significativamente a evolução dos doentes envolvidos. Desenvolvimento. Para divulgar as evidências científicas disponíveis no que se refere ao tratamento e prevenção do AVC, um comité ad hoc da Sociedade Ibero-americana de Doença Cérebro-vascular redigiu este guia, que recolhe de uma maneira esquemática todas aquelas recomendações vigentes com os níveis de evidência que as apoiam e o grau de recomendação para cada caso. Conclusões. Este documento pretende servir de apoio e guia para o tratamento e a prevenção nos doentes com AVC em todos os países ibero-americanos, e contribuir para alcançar os meios necessários para melhorar a atenção ao doente com AVC nas distintas administrações sanitárias. [REV NEUROL 2004; 39: 465-86]

Palavras chave. AVC. Doença cérebro-vascular. Guia. Protocolo. Recomendações. Sociedade Ibero-amerricana de Doença Cérebrovascular. 